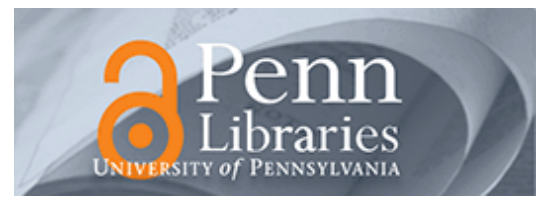

University of Pennsylvania ScholarlyCommons

October 2006

\title{
Minimizing Delay in Loss-Tolerant MAC Layer Multicast
}

\author{
Prasanna Chaporkar \\ University of Pennsylvania \\ Saswati Sarkar \\ University of Pennsylvania, swati@seas.upenn.edu
}

Follow this and additional works at: https://repository.upenn.edu/ese_papers

\section{Recommended Citation \\ Prasanna Chaporkar and Saswati Sarkar, "Minimizing Delay in Loss-Tolerant MAC Layer Multicast", . October 2006.}

Copyright 2006 IEEE. Reprinted from IEEE Transactions on Information Theory, Volume 51, Issue 10, October 2006, pages 4701-4713.

This material is posted here with permission of the IEEE. Such permission of the IEEE does not in any way imply IEEE endorsement of any of the University of Pennsylvania's products or services. Internal or personal use of this material is permitted. However, permission to reprint/republish this material for advertising or promotional purposes or for creating new collective works for resale or redistribution must be obtained from the IEEE by writing to pubs-permissions@ieee.org. By choosing to view this document, you agree to all provisions of the copyright laws protecting it.

This paper is posted at ScholarlyCommons. https://repository.upenn.edu/ese_papers/211

For more information, please contact repository@pobox.upenn.edu. 


\title{
Minimizing Delay in Loss-Tolerant MAC Layer Multicast
}

\begin{abstract}
The goal of this correspondence is to minimize delay in real-time multiple-access channel (MAC) layer multicast by exploiting the broadcast nature of wireless medium and limited loss tolerance of the applications. Multiple transmissions of a packet at the MAC layer significantly reduces the delay than that when only one transmission is allowed. But each additional transmission consumes additional power and increases network load. Therefore, the goal is to design a policy that judiciously uses the limited transmission opportunities so as to deliver each packet in the minimum possible time to the required number of group members. The problem is an instance of the stochastic shortest path problem, and using this formulation computationally simple, closed-form transmission strategies have been obtained in important special cases.

\section{Keywords}

broadcast property, dynamic programming, threshold policies, wireless multicast

\section{Comments}

Copyright 2006 IEEE. Reprinted from IEEE Transactions on Information Theory, Volume 51, Issue 10, October 2006, pages 4701-4713.

This material is posted here with permission of the IEEE. Such permission of the IEEE does not in any way imply IEEE endorsement of any of the University of Pennsylvania's products or services. Internal or personal use of this material is permitted. However, permission to reprint/republish this material for advertising or promotional purposes or for creating new collective works for resale or redistribution must be obtained from the IEEE by writing to pubs-permissions@ieee.org. By choosing to view this document, you agree to all provisions of the copyright laws protecting it.
\end{abstract}


$\pm 2^{k+1}$ and $S(a)$ is an integer, only two of the four sign combinations are possible, leading to $S(a)=-2^{k}$ or $S(a)=2^{k}$.

The preceding three cases give in total the four possible values $0, \pm 2^{k},-2^{k+1}$ for $S(a)$. Suppose the cross-correlation function $C_{d}(\tau)+1$ takes on the value zero $r$ times, the value $2^{k}$ is taken on $s$ times, the value $-2^{k}$ occurs $t$ times, and the value $-2^{k+1}$ occurs $v$ times. From Lemmas 1 and 2, it follows that

$$
\begin{aligned}
r+s+t+v & =2^{k}-1 \\
s-t-2 v & =1 \\
s+t+4 v & =2^{k}-1 .
\end{aligned}
$$

Since $S(a)= \pm 2^{k}$ is only possible in Case $2 \mathrm{c}$, which occurs $\left(2^{k}+\right.$ $1) / 3$ times, we get $s+t=\left(2^{k}+1\right) / 3$. The last equation leads to $v=$ $\left(2^{k-1}-1\right) / 3$ and therefore the first equation implies $r=2^{k-1}-1$. Finally, the last two equations give $t=0$ and $s=\left(2^{k}+1\right) / 3$.

\section{CONCLUSION}

We have found a new pair of $m$-sequences of different periods $2^{m}-1$ and $2^{k}-1$ where $m=2 k$ with three-valued cross correlation and we have completely determined the cross-correlation distribution. The pair of sequences differ by the sequences in the Kasami family in that we replace the decimation $d=1$ by the decimation $d=2^{l}-1$ where $k$ is an odd integer and $l=(k+1) / 2$.

\section{ACKNOWLEDGMENT}

The authors want to thank Alexander Kholosha for valuable comments.

\section{REFERENCES}

[1] H. Dobbertin, P. Felke, T. Helleseth, and P. Rosendahl, "Niho type cross-correlation functions via Dickson polynomials and Kloosterman sums," IEEE Trans. Inf. Theory, vol. 52, no. 2, pp. 613-627, Feb. 2006.

[2] H. Dobbertin, "Kasami power functions, permutation polynomials and cyclic difference sets type," in Difference Sets, Sequences, and Their Correlation Properties (Bad Windsheim, 1998). Dordrecht, The Netherlands: Kluwer, 1999, vol. 542, NATO Adv. Sci. Inst. Ser. C: Math. Phys. Sci., pp. 579-592.

[3] T. Helleseth, "Some results about the cross-correlation function between two maximal-linear sequences," Discr. Math., vol. 16, pp. 209-232, 1976.

[4] T. Helleseth and P. V. Kumar, "Sequences with low correlations," in Handbook in Coding Theory, V. S. Pless and W.C. Huffman, Eds. Amsterdam, The Netherlands: North-Holland, 1998, ch. 21.

[5] G. J. Ness and T. Helleseth, "Cross correlation of $m$-sequences of different lengths," IEEE Trans. Inf. Theory, vol. 52, no. 4, pp. 1637-1648, Apr. 2006.

[6] T. Kasami, Weight Distribution Formula for Some Classes of Cyclic Codes Univ. Illinois at Ubana-Champaign, Coordinated Science Lab., Urbana, IL, 1966, Tech. Rep. R-285 (AD 637524).

\section{Minimizing Delay in Loss-Tolerant MAC Layer Multicast}

Prasanna Chaporkar and Saswati Sarkar, Member, IEEE

\begin{abstract}
The goal of this correspondence is to minimize delay in real-time multiple-access channel (MAC) layer multicast by exploiting the broadcast nature of wireless medium and limited loss tolerance of the applications. Multiple transmissions of a packet at the MAC layer significantly reduces the delay than that when only one transmission is allowed. But each additional transmission consumes additional power and increases network load. Therefore, the goal is to design a policy that judiciously uses the limited transmission opportunities so as to deliver each packet in the minimum possible time to the required number of group members. The problem is an instance of the stochastic shortest path problem, and using this formulation computationally simple, closed-form transmission strategies have been obtained in important special cases.
\end{abstract}

Index Terms-Broadcast property, dynamic programming, threshold policies, wireless multicast.

\section{INTRODUCTION}

In wireless networks, many real-time applications such as conference meetings, emergency operation in case of a natural disaster, and military operations require one to many (multicast) communication. Real-time applications can tolerate some packet loss but require low delay. Our contribution is to develop transmission schemes that minimize the delay in real-time medium access control (MAC) layer multicast by exploiting the limited loss tolerance and the broadcast property of wireless medium. Most of the work in wireless multicast has focused on the network and transport layers, e.g., [5], [8], [12]. Though the performance of the network and transport layer protocols depends on the efficiency of the MAC layer strategy, MAC layer multicast has not been adequately explored. Our work is directed towards filling this void.

Now, we describe the challenges in minimizing the delay attained by MAC layer multicast schemes. Due to the broadcast property of wireless communication, a sender can deliver a packet to all its receivers which are in its transmission range using a single transmission. Apparently, this broadcast nature can be used to reduce the delay at the MAC layer. But, the broadcast nature also introduces critical challenges. A multicast specific challenge is that some but not all the receivers may be ready to receive due to the interference in their neighborhood and transmission quality in wireless channels. Consider a MAC layer multicast session from a sender $S_{1}$ to receivers $R_{1}$ to $R_{4}$ which are in $S_{1}$ 's transmission range (Fig. 1). When $S_{2}$ is transmitting, $R_{1}$ and $R_{2}$ cannot receive a transmission from $S_{1}$ as both the transmissions will collide at these receivers. However, $R_{3}$ and $R_{4}$ can still receive the transmission

Manuscript received August 17, 2005; revised April 12, 2006. This work was supported by the National Science Foundation under Grants ANI-0106984, NCR-0238340 and CNS-0435306. The material in this correspondence was presented in part at the 3rd International Symposium on Modeling and Optimization in Mobile, Ad Hoc, and Wireless Networks, Riva Del Garda, Italy, April 2005, and at the 41st Annual Allerton Conference on Communications, Control and Computing, Monticello, IL, October 2003.

P. Chaporkar is with INRIA, Domaine de Voluceau, Rocquencourt, 78153 Le Chesnay Cedex, France (e-mail: Prasanna.Chaporkar@ens.fr).

S. Sarkar is with the Department of Electrical and Systems Engineering, University of Pennsylvania, Philadelphia PA 19104 USA (e-mail: swatit@seas.upenn.edu).

Communicated by E. Modiano, Associate Editor for Communication Networks.

Color versions of Figs. 1, 2, 8, and 12 are available online at http://ieeexplore. ieee.org.

Digital Object Identifier 10.1109/TIT.2006.881756 


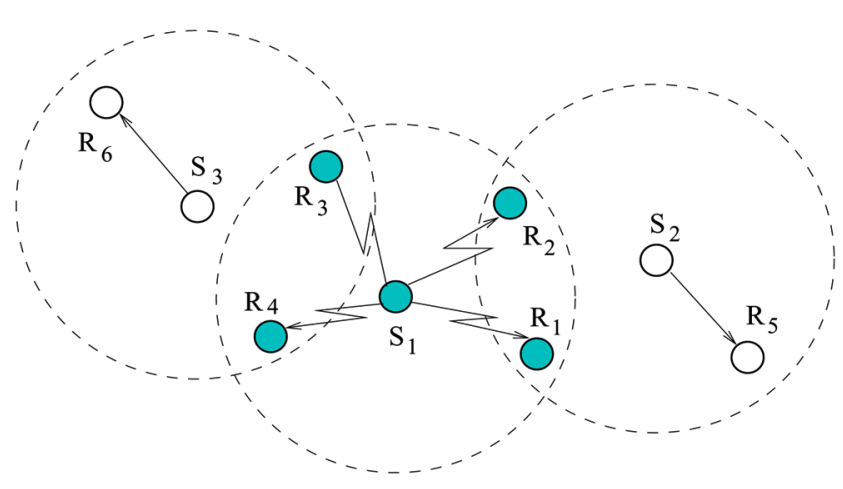

Fig. 1. An example to demonstrate the advantages and the challenges associated with wireless multicast. Here, $S_{1}, S_{2}$, and $S_{3}$ are senders and $R_{1}, \ldots, R_{6}$ are receivers. Dashed circles indicate the communication ranges of senders.

if $S_{3}$ is not transmitting. It is not clear whether the delay will be minimized if $S_{1}$ transmits the packet only when all the receivers are ready simultaneously, or if $S_{1}$ transmits separately to receivers $R_{1}-R_{2}$ and $R_{3}-R_{4}$. The following example illustrates the point.

Example: We compare the expected delay in delivering a Head of Line (HoL) packet for two transmission strategies in Fig. 1: a) $S_{1}$ transmits when all the receivers are ready, and b) $S_{1}$ transmits first to $R_{1}-R_{2}$ and then to $R_{3}-R_{4}$. Let $S_{2}$ and $S_{3}$ require one slot to transmit a packet. We assume that $S_{2}$ and $S_{3}$ do not transmit when $S_{1}$ is transmitting, and otherwise transmit independently with probability (w.p.) $1-p$ each. The expected delay under the first policy $\left(\boldsymbol{E}\left[D_{1}\right]\right)$ is equal to the expected time till all the four receivers become ready plus the packet transmission time which we denote as $V$, i.e., $\boldsymbol{E}\left[D_{1}\right]=1 / p^{2}+V$. The expected delay under the second policy $\left(\boldsymbol{E}\left[D_{2}\right]\right)$ is equal to the expected time till $R_{1}-R_{2}$ become ready plus the expected time till $R_{3}-R_{4}$ become ready plus $2 V$, i.e., $\boldsymbol{E}\left[D_{2}\right]=2(1 / p+V)$. Let $p=0.1$. Now, when $V=10$, then $E\left[D_{1}\right]=110$ slots and $\boldsymbol{E}\left[D_{2}\right]=40$ slots. When $V=100$, then $\boldsymbol{E}\left[D_{1}\right]=200$ slots and $\boldsymbol{E}\left[D_{2}\right]=220$ slots. Thus, different policies achieve smaller delays in different scenarios.

The preceding example shows that multiple transmissions, if utilized properly, can significantly reduce the delay. But multiple transmissions also increase the power consumption and the network load. Hence, it is desirable to limit the number of such transmissions. Thus, one needs to design a policy that judiciously utilizes the transmissions and the broadcast nature of wireless medium to minimize the delay.

Real-time applications can accommodate some packet loss without noticeable degradation in the quality of service, e.g., for voice, depending on the encoding and transmission schemes used, 20\% packet loss can be acceptable [7]. Hence, depending on the loss tolerance of applications, it may suffice to deliver each packet only to a certain fraction of the multicast group. The following example demonstrates that a small loss tolerance can significantly reduce the delay.

Example: Consider a MAC layer multicast session from $S_{1}$ to receivers $R_{1}, R_{2}, \ldots, R_{20}$. Let a receiver be ready in a slot w.p. 0.6 independent of its state in other slots and the states of other receivers in any slot. Each receiver must receiver $95 \%$ of the transmitted packets. We consider two policies: a) $S_{1}$ transmits only when all receivers are ready, and b) $S_{1}$ transmits when 19 or more receivers are ready. Let the transmission time for each packet be one slot. The mean delays under policies a) and b) are 27351.11 slots and 1908.22 slots, respectively. The expected fraction of packets lost at a receiver under policies a) and b) are 0 and 0.047 , respectively. Thus, the delay can be significantly reduced by exploiting the loss tolerance.
We investigate the tradeoff between loss and delay for MAC layer multicast. Specifically, we study the problem of minimizing the mean delay to deliver an HoL packet to $Z$ out of total $G$ receivers using at most $K$ transmissions. The parameters $Z$ and $K$ depend on loss tolerance of the application and power constraints, respectively. In Sections II and III, we describe the system model, and formulate the optimization goal as a stochastic shortest path (SSP) problem, respectively [2], [3]. The time and memory required by the SSP computation increases exponentially with increase in $G$. Next, using the SSP formulation, we show that the computation time and the storage requirements of the optimal policy are polynomial in $G, K, Z$ when the readiness states of different receivers constitute mutually independent and identically distributed Markov processes (Section IV-A). Next, we consider two extreme cases of the above independent and identically distributed (i.i.d.) Markovian receiver readiness process: the readiness process of each receiver is 1) bursty, i.e., the transition probabilities of the MC are small (Section IV-B), and 2) Bernoulli (Section IV-C). In both these cases, we prove that the optimal policy is threshold-type, and the storage requirements and the computation time for the optimal thresholds are polynomial in $G, K, Z$. In Section V, we discuss several salient features of the policies and evaluate their performances numerically and using simulations. We present all proofs in the Appendix.

We briefly review the MAC protocols for multicast in ad hoc networks. IEEE 802.11 supports MAC layer multicast by disabling the control mesage exchange and broadcasting the data packets; the protocol is not therefore reliable. Tang et al. have proposed to enhance the reliability of IEEE 802.11 by a) using the capture mechanism to ensure that at least one receiver is ready when the packet is broadcast [10], and b) by transmitting a packet to each receiver separately in unicast mode [11]. The first scheme may not provide desired loss rates, and the second scheme does not exploit the broadcast property.

\section{SYSTEM MODEL}

We consider a single multicast session with $G$ receivers. The impact of the network and the channel errors on the multicast session is that the receivers are not always ready to receive. This may happen because of a transmission in the neighborhood of a receiver, bursty channel errors, or power-saving operation of a receiver. Thus, the receiver readiness states are correlated in the same time slot, and across the time slots. We model the readiness process of all the receivers as a Markov chain (MC) with an arbitrary transition probability matrix (TPM) $\widehat{\boldsymbol{B}}$. A state of the MC is the $G$-dimensional readiness vector $\vec{j}=\left[\begin{array}{llll}j_{1} & j_{2} & \cdots & j_{G}\end{array}\right]$, where the component $j_{l}$ is 1 if the $l$ th receiver is ready and 0 otherwise. Let $\mathcal{C}$ denote the state space of the MC. We assume that the $2^{G} \times 2^{G}$ TPM $\widehat{\boldsymbol{B}}$ is irreducible, aperiodic, and time-homogeneous. We adopt this model because in a distributed environment the senders do not coordinate their transmissions, and only observe the readiness states of their receivers. Thus, from the perspective of a sender, the network is a stochastic disturbance which is not controllable but only partially observable. The arbitrary Markovian transitions of the readiness process allow us to consider different network loads and different inter-session interactions.

A sender queries the readiness states of the receivers by transmitting control packets, and decides whether to transmit a packet depending on the transmission strategy and result of the query. Every receiver maintains its readiness state throughout the transmission. This assumption is justified because the time scale of a change of transmission quality is much larger than the duration of packet transmission. Also, the level of interference does not change during a packet transmission, since in several MAC protocols (e.g., IEEE 802.11), the exchange of control messages prevents a new transmission during an ongoing transmission in the reception range of the receiver. The sender backs off for a random 


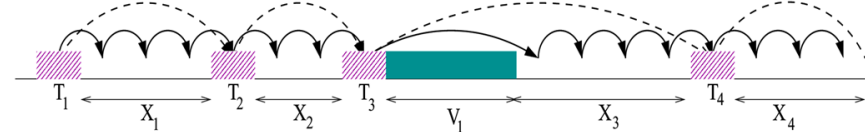

Fig. 2. Typical transitions of the receiver readiness process (solid arcs) and the sampled readiness process (dashed arcs). A box indicates a time slot. The $T_{i}$ 's, $V_{i}$ 's, and $X_{i}$ 's denote the sample points, duration of transmission, and duration of backoff, respectively.

duration before querying the system again, irrespective of the transmission decision, so as to allow other senders to use the shared medium. The structure of the multiple-access protocol described above is similar to IEEE 802.11. Note that the receiver readiness process is Markovian only when restricted to the slots in which the sender queries or backs off, e.g., in duration $\left[T_{1}, T_{3}\right] \cup X_{3} \cup T_{4} \cup X_{4}$ in Fig. 2.

We assume that time is slotted. The packet transmission times and backoff durations are i.i.d. random variables with arbitrary probability distributions and finite expected values $\boldsymbol{E}[V]$ and $\boldsymbol{E}[X]$, respectively. For brevity, let $\bar{X}=\boldsymbol{E}[X]+1$ and $\bar{V}=\boldsymbol{E}[V+X]+1$. The slots in which the sender queries the readiness states are called sample points, and the readiness process observed by the sender is called sampled readiness process. Note that the sampled readiness process is also an irreducible, aperiodic and time-homogeneous MC. Let $\boldsymbol{B}$ denote the TPM for the sampled readiness process. Then, the transition probabilities of the sampled readiness process are

$$
B_{\vec{j}, \vec{j}_{1}}=\sum_{l=1}^{\infty} \widehat{B}_{\vec{j}, \vec{j}_{1}}^{(l)} \boldsymbol{P}\{X=l-1\}
$$

where $\widehat{B}_{\vec{j}, \vec{j}_{1}}^{(l)}$ is the probability of being in state $\vec{j}_{1}$ starting from state $\vec{j}$ after $l$ transitions of the original readiness process. At any time, a receiver is satisfied if it has received the packet in prior transmissions; otherwise, it is unsatisfied. Initially, every receiver is unsatisfied and with subsequent transmissions some receivers become satisfied.

\section{A FRAMEWORK FOR COMPUTING THE OPTIMAL TRANSMISSION POLICY}

Our goal is to design a transmission strategy that minimizes the expected time to deliver an HoL packet to at least $Z$ receivers using at most $K$ transmissions. This optimization can be formulated as a stochastic shortest path problem (SSP) as follows. Let $\vec{a}=\left[\begin{array}{llll}a_{1} & a_{2} & \cdots & a_{G}\end{array}\right]$ where $a_{i}$ is 1 if receiver $i$ is satisfied, and $a_{i}$ is 0 otherwise. The system state is the vector $(k, \vec{a}, \vec{j})$, where $k$ is the number of completed transmissions and $\vec{j}$ is the readiness vector. Note that when $k=0$, then $\vec{a}=\overrightarrow{0}$, where $\overrightarrow{0}$ is the $G$-dimensional vector of zeros. In every state $(k, \vec{a}, \vec{j})$, the sender can either back off or transmit. If the sender backs off, then the state becomes $\left(k, \vec{a}, \vec{j}_{1}\right)$ w.p. $B_{\vec{j}} \vec{j}_{1}$. If the sender transmits, then the state becomes $\left(k+1, \vec{j} \circ \vec{a}, \vec{j}_{1}\right)$ w.p. $B_{\vec{j}}, \vec{j}_{1}$, where $\vec{j} \circ \vec{a}$ denotes the element-wise OR operation of the vectors $\vec{j}$ and $\vec{a}$. The process terminates when $Z$ or more receivers are satisfied, i.e., the states $(k, \vec{a}, \vec{j})$ such that $\sum_{i=1}^{G} a_{i} \geq Z$ are the termination states. The system needs to reach a termination state in the minimum expected time. Let $J^{*}(k, \vec{a}, \vec{j})$ denote the minimum expected time to terminate (minimum termination time) from $(k, \vec{a}, \vec{j})$. Clearly, for every termination state $(k, \vec{a}, \vec{j})$ (states with $\left.\sum_{i=1}^{G} a_{i} \geq Z\right), J^{*}(k, z, t)=0$. If after $K-1$ transmissions the number of satisfied receivers $z$ is less than $Z$, then the sender transmits only if $Z-z$ or more unsatisfied receivers are ready. Thus, the process always terminates. Also,

$$
J^{*}(K-1, \vec{a}, \vec{j})=\bar{V}+\tilde{s}_{\vec{j}, \mathcal{J}_{\vec{a}}}, \quad \text { if } \sum_{i=1}^{G} a_{i}<Z
$$

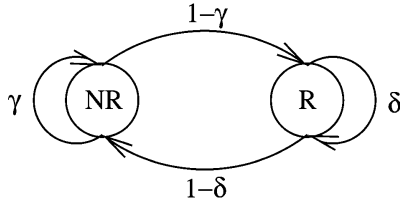

Fig. 3. The readiness process for a receiver when the readiness processes are i.i.d. Markovian. The readiness process is as observed at the sample points. $N R$ and $R$ indicates states Not Ready and Ready, respectively.

where

$$
\mathcal{J}_{\vec{a}}=\left\{\vec{j}: \sum_{i=1}^{G} a_{i} \circ j_{i} \geq Z\right\}
$$

and $\tilde{s}_{\vec{j}, \mathcal{J}_{\vec{a}}}$ denote the product of $\bar{X}$ and the expected number of sample points required to reach any of the states $\vec{j}_{1} \in \mathcal{J}_{\vec{a}}$ for the first time starting from $\vec{j}$ in the receiver readiness process.

Let $J_{T}^{*}(k, \vec{a}, \vec{j})$ and $J_{B}^{*}(k, \vec{a}, \vec{j})$ denote the minimum expected termination time from $(k, \vec{a}, \vec{j})$ if the control decision is to transmit and back off, respectively. For convenience, we assume that $J^{*}(K, \vec{a}, \vec{j})=\infty$ if $\sum_{i=1}^{G} a_{i}<Z$; note that the system never reaches these states. The minimum expected termination times from the states with $\sum_{i=1}^{G} a_{i}<Z$, $k \leq K-1$, satisfy the following Bellman's equations:

$$
\begin{aligned}
& J^{*}(k, \vec{a}, \vec{j})=\min \left\{J_{T}^{*}(k, \vec{a}, \vec{j}), J_{B}^{*}(k, \vec{a}, \vec{j})\right\} \\
& J_{T}^{*}(k, \vec{a}, \vec{j})=\bar{V}+\sum_{\vec{j}_{1} \in \mathcal{C}} B_{\vec{j}, \vec{j}_{1}} J^{*}\left(k+1, \vec{j} \circ \vec{a}, \vec{j}_{1}\right) \\
& J_{B}^{*}(k, \vec{a}, \vec{j})=\bar{X}+\sum_{\vec{j}_{1} \in \mathcal{C}} B_{\vec{j}_{j}, \vec{j}_{1}} J^{*}\left(k, \vec{a}, \vec{j}_{1}\right) .
\end{aligned}
$$

If $J^{*}(k, \vec{a}, \vec{j})=J_{B}^{*}(k, \vec{a}, \vec{j})$, then the optimal decision in state $(k, \vec{a}, \vec{j})$ is to back off; otherwise, the optimal decision is to transmit. Thus, the optimal strategy can be obtained by solving the Bellman's equations (2)-(4).

Bellman's equations can be solved using several standard methods, among which the Linear Programming method [3] has the least complexity. In this method, we need to solve a linear program in which the number of variables and constraints are of the order of the number of system states which is $K 2^{2 G}$ in this case. Thus, the complexity of this method is $O\left(\left(K 2^{2 G}\right)^{3.5}\right)$ [6]. Once the optimal policy is computed, online transmission decisions can be made using a lookup table which needs to store all $O\left(K 2^{2 G}\right)$ system states. Thus, both the time and the memory required for computing and executing the optimal strategy increases exponentially with increase in $G$.

\section{Optimal Transmission StRategies In SPEcial Cases}

We now consider the special case that the receiver readiness states evolve as per i.i.d. Markovian readiness processes. Specifically, each receiver's readiness process at the sample points evolves as per a twostate Markov process (Fig. 3), which changes state from ready (not ready) to not ready (ready) with probability $1-\delta(1-\gamma)$. The readiness states of different receivers are mutually independent and identically distributed. We obtain an optimal policy whose computational complexity is $O\left(K G^{7}\right)$ and memory requirement is $O\left(K G^{2}\right)$; both time and memory requirements therefore increase polynomially with increase in $K$ and $G$ (Section IV-A).

We next consider two extreme scenarios of i.i.d. Markovian readiness processes: a) bursty readiness process $(1-\gamma$ and $1-\delta \approx 0)$, and b) Bernoulli readiness process $(1-\gamma=\delta)$. We prove in Sections IV-B and $\mathrm{C}$ that in both these extreme cases, the optimal strategies are 


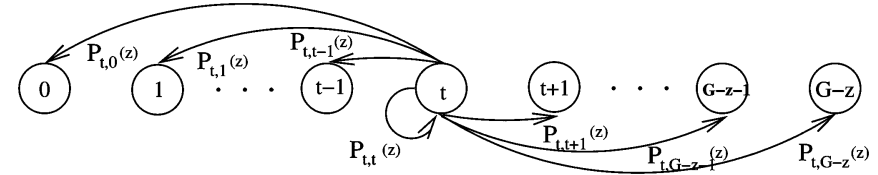

Fig. 4. Possible transitions from state $t$ in the aggregate readiness process of $G-z$ unsatisfied receivers.

threshold-type ${ }^{1}$ and have lower computation time $\left(O\left(K G^{3}\right)\right.$ for bursty readiness process, and $O\left(K G^{2}\right)$ for Bernoulli readiness process) and lower memory requirements $(O(K G))$ than that for arbitrary i.i.d. Markovian readiness processes. In threshold-type transmission policies, before each transmission, the sender selects a threshold and transmits only when the number of unsatisfied ready receivers exceeds the selected threshold. We show that the optimal threshold for each transmission depends on the number of transmissions utilized so far and the number of receivers that have already received the packet, and can be computed in $O\left(K G^{3}\right)$ and $O\left(K G^{2}\right)$ times for bursty and Bernoulli readiness processes, respectively.

\section{A. I.I.D. Markovian}

We now consider i.i.d. Markovian readiness processes. Since the readiness states are i.i.d., intuitively, the expected time for termination does not depend on identity of the satisfied or unsatisfied receivers, but rather depends only on the number of satisfied receivers $(z)$ and the number of unsatisfied ready receivers $(t)$. We prove this formally in the following lemma. Let $z_{\vec{a}}$ denote the number of satisfied receivers, and let $t_{\vec{a}, \vec{j}}$ denote the number of unsatisfied ready receivers in state $(k, \vec{a}, \vec{j})$, i.e.,

$$
z_{\vec{a}}=\sum_{i=1}^{G} a_{i} \text { and } t_{\vec{a}, \vec{j}}=\sum_{i=1}^{G} \max \left\{j_{i}-a_{i}, 0\right\} .
$$

Lemma 1: Let in system states $(k, \vec{a}, \vec{j})$ and $\left(k, \vec{a}_{1}, \vec{j}_{1}\right)$

$$
z_{\vec{a}}=z_{\vec{a}_{1}} \text { and } t_{\vec{a}, \vec{j}}=t_{\vec{a}_{1}, \vec{j}_{1}} \text {. }
$$

Then, for an i.i.d. Markovian receiver readiness process

$$
J^{*}(k, \vec{a}, \vec{j})=J^{*}\left(k, \vec{a}_{1}, \vec{j}_{1}\right), \quad \text { for every } k \in\{0, \ldots, K\} .
$$

From Lemma 1, it suffices to consider the system state as $(k, z, t)$. Thus, the number of system states is $O\left(K G^{2}\right)$. Since in each state there are only two possible actions (transmission and backoff), the memory required for storing the optimal policy is also $O\left(K G^{2}\right)$.

Another important consequence of Lemma 1 is that the expected termination time depends on the initial readiness vector only through the initial number of ready receivers. Hence, when the initial number of ready receivers is $t$, we refer to the problem of minimizing the above expected time as $\mathcal{P}(K, G, Z, t)$. We now describe how $\mathcal{P}(K, G, Z, t)$ can be solved in polynomial time.

We now consider the aggregate readiness process of receivers. The state of the aggregate readiness process is the number of ready receivers (Fig. 4). Clearly, the aggregate readiness process is a Markov process. The transition probability in the aggregate readiness process of $G-z$ receivers, $P_{t, t_{1}}(z)$, denotes the probability that $t_{1}$ receivers are ready at

${ }^{1}$ For bursty readiness processes, we have proved that the optimal strategy is threshold-type except at the first transmission of the packet in which the optimal policy can transmit in one of two states. the current sample point given that $t$ out of $G-z$ receivers were ready at the previous sample point. Then, for all $t, t_{1} \in\{0, \ldots, G-z\}$

$$
\begin{aligned}
P_{t, t_{1}}(z)=\sum_{u=0}^{t}\left(\begin{array}{l}
t \\
u
\end{array}\right) \delta^{u}(1-\delta)^{t-u} \\
\\
\quad \times\left(\begin{array}{c}
G-z-t \\
t_{1}-u
\end{array}\right)(1-\gamma)^{t_{1}-u} \gamma^{G-z-t-t_{1}+u}
\end{aligned}
$$

Here, $\left(\begin{array}{c}\hat{c} \\ c\end{array}\right)=0$ if $\hat{c}<0$ or $\widehat{c}>c$. If the sender backs off in state $(k, z, t)$, then the state changes to $\left(k, z, t_{1}\right)$ w.p. $P_{t, t_{1}}(z)$, and if the sender transmits in state $(k, z, t)$, then the state changes to $(k+1, z+$ $\left.t, t_{1}\right)$ w.p. $P_{0, t_{1}}(z+t)$.

The policy $\pi(K, Z)$ comprising of control decisions $\left\{D_{k, z, t}, k=\right.$ $0, \ldots, K-1, z=0, \ldots, Z-1, t=0, \ldots G-z\}$ computed in Fig. 5 solves $\mathcal{P}(K, G, Z, t)$ for all $t$. The algorithm in Fig. 5 first solves $\mathcal{P}\left(1, G-z_{1}, Z-z_{1}, t_{1}\right)$ for all $z_{1}, t_{1}$ using (1). Subsequently, it progressively solves $\mathcal{P}\left(k, G-z_{1}, Z-z_{1}, t_{1}\right)$ for all $z_{1}, t_{1}$ and $k=2,3, \ldots K$ by solving the linear program LP1 in Fig. 5. Now, (B3) in Fig. 5 obtains the optimal decisions in every state $(k, z, t)$ as the optimal decision $D_{k, z, t}$ is to transmit (back off, respectively) in state $(k, z, t)$ if and only if $J_{T}^{*}(k, z, t)<(\geq$, resp. $) J_{B}^{*}(k, z, t)$.

Theorem 1: For every $k \leq K-1$ and $z, t, \widehat{J}^{*}(k, z, t)=J^{*}(k, z, t)$. For every $t=0, \ldots, G, \pi(K, Z)$ solves $\mathcal{P}(K, G, Z, t)$

Finally, $\pi(K, Z)$ is computed in Fig. 5 by solving $O(K)$ linear programs each with $O\left(G^{2}\right)$ variables and constraints. Thus, $\pi(K, Z)$ can be computed in $O\left(K G^{7}\right)$ time [6].

\section{B. Subcase 1: Bursty Receiver Readiness States}

We consider a special case of i.i.d. Markovian readiness process in which the receiver readiness states are bursty, i.e., the transition probabilities $1-\delta$ and $1-\gamma$ are close to zero. From (7), we observe that if $|t-u| \geq 2$, then $P_{t, u}(z) \approx 0$ as it only contains terms with higher powers of $(1-\delta)$ and/or $(1-\gamma)$ for every $z \in\{0, \ldots, G\}$. Now, for $u \in\{t-1, t, t+1\}$ and $t \in\{0, \ldots, G-z\}$

$$
\begin{aligned}
P_{t, t+1}(z) & \stackrel{\text { Def }}{=} \alpha_{t}(z) \approx(G-t-z) \delta^{t} \gamma^{G-z-t-1}(1-\gamma) \\
P_{t, t-1}(z) & \stackrel{\text { Def }}{=} \beta_{t}(z) \approx t \gamma^{G-z-t} \delta^{t-1}(1-\delta) \\
P_{t, t}(z) & \approx 1-\left(\alpha_{t}(z)+\beta_{t}(z)\right)
\end{aligned}
$$

Thus, the aggregate receiver readiness process can be approximated as a nonhomogeneous birth-death (BD) process (Fig. 6). Let $t_{k}$ be the number of ready, unsatisfied receivers right after the $k$ th transmission, for $k \geq 1$. Let $t_{0}$ be the number of ready receivers when the packet reaches the $\mathrm{HoL}$ position. Using the $\mathrm{BD}$ approximation, we obtain a closed-form computationally simple optimal transmission strategy, $\pi_{1}(K, Z)$ (Fig. 7). We prove that the optimal transmission decision in any state $(k, z, t)$ is to transmit if and only if i) $t$ is greater than or equal to a threshold $\tau(k, z)$, if $k \geq 1$ and ii) $t$ has one of two values that depend on $t_{0}$, if $k=0$. Thus, the optimal policy can be stored as a function of $k$ and another variable ( $z$ or $t_{0}$ ) that has $G+1$ possible values; this requires $O(K G)$ memory.

We first explain why the transmission policy at $k=0$ differs from that at other values of $k$. Note that $t_{0} \in\{0, \ldots, G\}$, while $t_{k} \in\{0,1\}$, for $k \geq 1$. Let $z$ receivers be satisfied after $k$ transmissions and let $\mathcal{T}_{k, z}$ denote the set of aggregate readiness states in which the optimal decision is to transmit

$$
\mathcal{T}_{k, z}=\left\{t: 0 \leq u \leq G-z \text { and } J_{T}^{*}(k, z, t)<J_{B}^{*}(k, z, t)\right\} .
$$

Let $m_{k, z}$ denote the smallest member of $\mathcal{T}_{k, z}$. Clearly, $m_{k, z} \geq 1$ for every $k$ and $z$. Let $k \geq 1$. Since $t_{k} \in\{0,1\}, t_{k} \leq m_{k, z}$. Thus, 


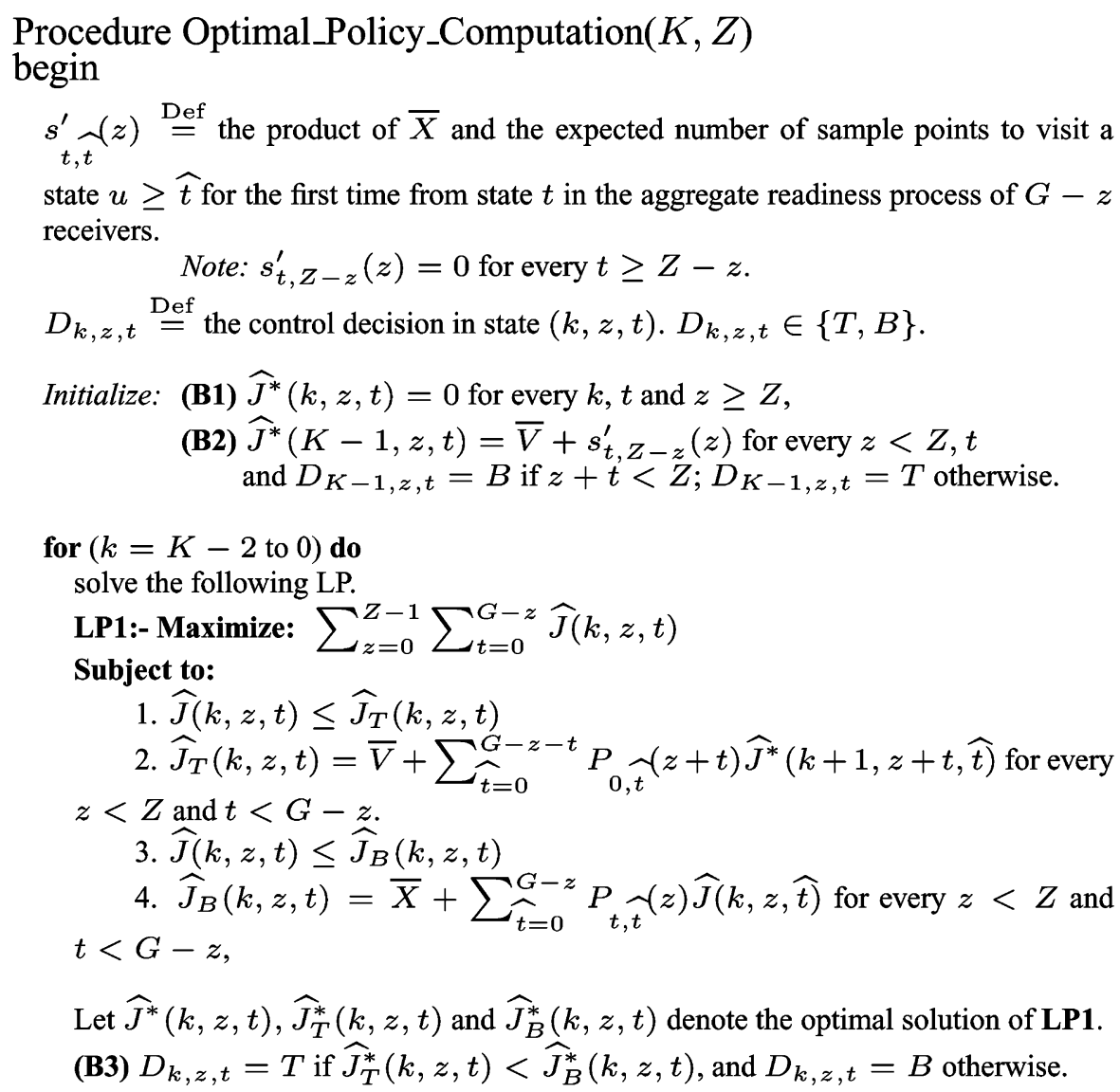

Policy $\pi(K, Z)$ transmits in state $(k, z, t)$ if $D_{k, z, t}=T$; it backs off otherwise. end

Fig. 5. Pseudocode for the optimal transmission policy, $\pi(K, Z)$, when the receiver readiness processes are i.i.d. Markovian.

due to its $\mathrm{BD}$ nature, starting from $t_{k}$, the aggregate readiness process of unsatisfied receivers cannot reach states greater than $m_{k, z}+1$ before $m_{k, z}$. Thus, the optimal policy transmits when $m_{k, z}$ unsatisfied receivers are ready (see Fig. 8(a)). This explains the existence of optimal thresholds in this case. But, $t_{0}$ can exceed 1 . Hence, $m_{0, z}$ may be less than $t_{0}$. Thus, the optimal policy transmits when either $u_{1}$ or $u_{2}$ unsatisfied receivers are ready, where $u_{1}$ is the largest element of $\mathcal{T}_{0, z}$ such that $u_{1}<t_{0}$, and $u_{2}$ is the smallest element of $\mathcal{T}_{0, z}$ such that $u_{2}>t_{0}$ (see Fig. 8(b)). Thus, for $k=0$, the optimal strategy may not be threshold-type, and the minimum expected termination time is the minimum of the expected termination times in the cases that $m_{0, z} \geq t_{0}\left(J_{1}(0,0, t)\right)$ and $m_{0, z}<t_{0}\left(J_{2}(0,0, t)\right)$ (Fig. 7).

$\pi_{1}(K, Z)$ in Fig. 7 first computes $\widehat{J}^{*}\left(K-1, z, t_{K-1}\right)$ for $z \leq G$, $t_{K-1} \in\{0,1\}$, and the optimal threshold $\tau(K-1, z)$ from (1) and the $\mathrm{BD}$ nature of the aggregate readiness process $((\mathbf{C 1})$ and $(\mathbf{C 2}))$. Subsequently, it sequentially computes, $\widehat{J}^{*}\left(k, z, t_{k}\right)$ for $z \leq G, t_{k} \in\{0,1\}$, and the optimal threshold $\tau(k, z)$ for $k=K-2, K-3, \ldots, 1$ $((\mathbf{C 3}),(\mathbf{C 4})$, and $(\mathbf{C 5}))$. Finally, it computes $\widehat{J}^{*}\left(0, z, t_{0}\right)$ for $z \leq G$, $t_{0} \in\{0, \ldots, G\}$, and the possible transmission states $\tau\left(t_{0}\right)((\mathbf{C 6})$ and $(\mathbf{C} 7))$. We prove that these $\widehat{J}^{*}(k, z, t)$ equal corresponding values of $J^{*}(k, z, t)$ (Appendix B). $\pi_{1}(K, Z)$ can be computed in $O\left(K G^{3}\right)$ time.

Theorem 2: Let the aggregate receiver readiness processes of unsatisfied receivers be a BD process. Then, $\pi_{1}(K, Z)$ solves $\mathcal{P}(K, G, Z, t)$ for every $t \in\{0, \ldots, G\}$.
Note that the BD modeling is an approximation for i.i.d. Markovian processes for low values of $1-\gamma$ and $1-\delta$. We now evaluate the error due to this approximation. Let $D_{O}$ denote the minimum expected delay obtained using the policy in Fig. 5. Let $D_{A}$ denote the expected delay obtained using the policy in Fig. 7. In Fig. 9, we plot the percentage normalized approximation error $\frac{D_{A}-D_{O}}{D_{O}} \times 100$ as a function of $1-\delta$. This normalized approximation error turns out to be 0 for small values of $1-\delta$ and it is less than $2 \%$ for $1-\delta \leq 0.3$. This validates the BD approximation. Note that when the readiness states are generated by a Raleigh-fading channel which is good for $99 \%$ of the time and has mean fade duration of 10 slots, then $1-\delta=0.001$ and $1-\gamma=0.1$ [9].

\section{Subcase 2: Bernoulli Readiness States}

Now, we assume that the receiver readiness states are i.i.d. Bernoulli, i.e., in a slot, a receiver is ready w.p. $p$. Now, $P_{t_{1}, t_{2}}(z)$ does not depend on $t_{1}$ as the readiness states are independent across the slots. Thus, it suffices to maintain a two-dimensional system state $(k, z)$ and hence the memory required for executing the optimal policy is $O(K G)$. Also, now the aggregate readiness process can have transitions to nonadjacent states (Fig. 4). The optimal transmission algorithm $\pi_{2}(K, Z)$ (Fig. 10) is, however, still threshold type, and can be computed in $O\left(K G^{2}\right)$ time.

Theorem 3: For i.i.d. Bernoulli receiver readiness processes $\pi_{2}(K, Z)$ solves $\mathcal{P}(K, G, Z, t)$ for every $t \in\{0, \ldots, G\}$. 


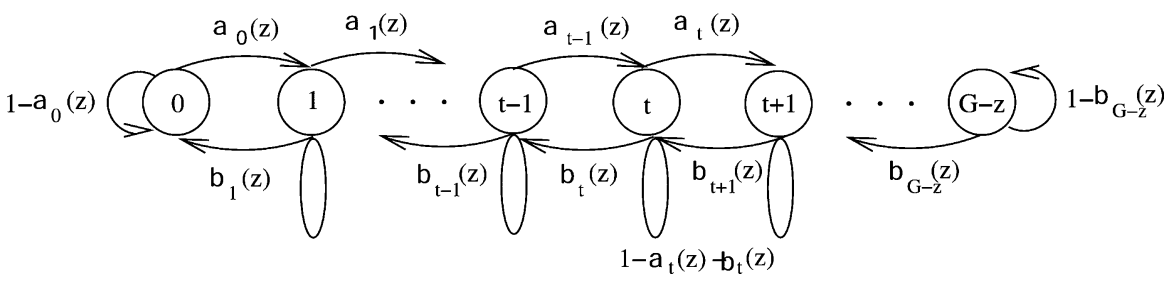

Fig. 6. A BD process that approximates the aggregate receiver readiness process of $G-z$ unsatisfied receivers when the receivers have bursty i.i.d. Markovian readiness processes.

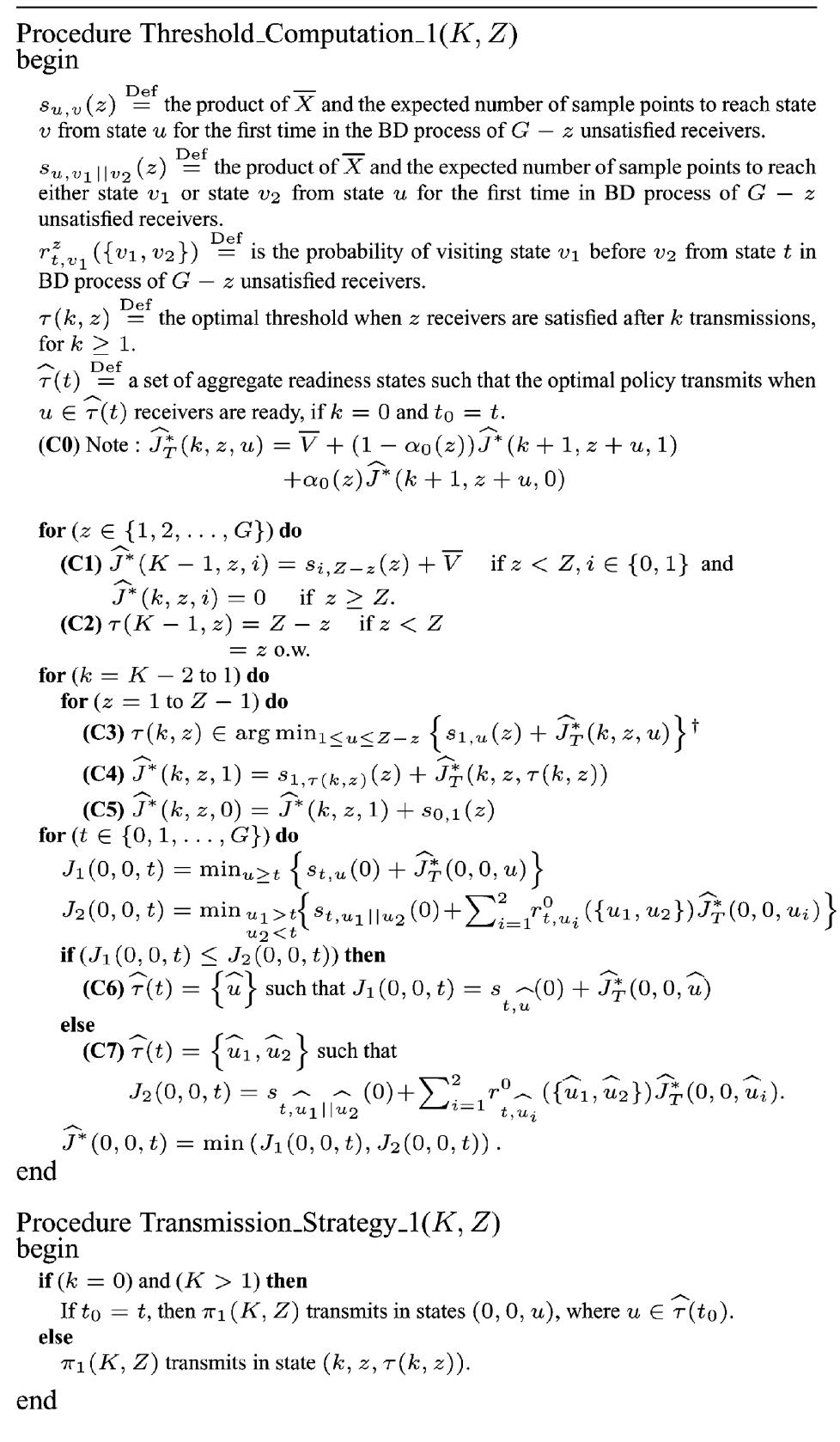

Fig. 7. Pseudocode for the optimal transmission policy, $\pi_{1}(K, Z)$, when the receiver readiness processes are i.i.d. bursty Markovian.

\section{Performance Evaluation AND Discussion}

We first discuss how $Z$ can be chosen based on application requirements. The loss at a receiver is the fraction of packets transmitted by the sender which the receiver does not receive, and the system loss is the sum of the losses of the receivers. Usually, higher layer applications and coding schemes (e.g., digital fountain [4]) require that the loss at each receiver be upper-bounded by a constant. In several cases, 
(a)

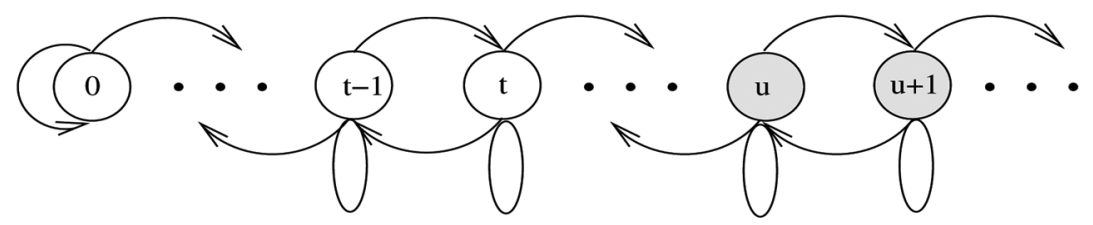

(b)

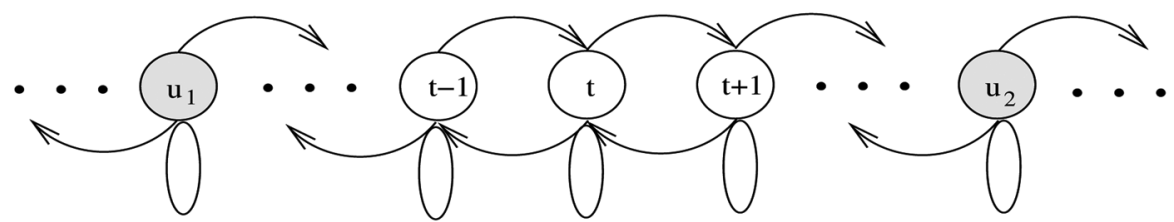

Fig. 8. The aggregate readiness process of unsatisfied receivers. The shaded states are in $\mathcal{T}_{k, z}$. Let the process be in state $t$. In case a), $m_{k, z}=u>t$, and in case b) $m_{k, z} \leq u_{1}<t$. The optimal policy transmits when (a) $u$ unsatisfied receivers are ready in case a), and (b) when either $u_{1}$ or $u_{2}$ receivers are ready in case b).

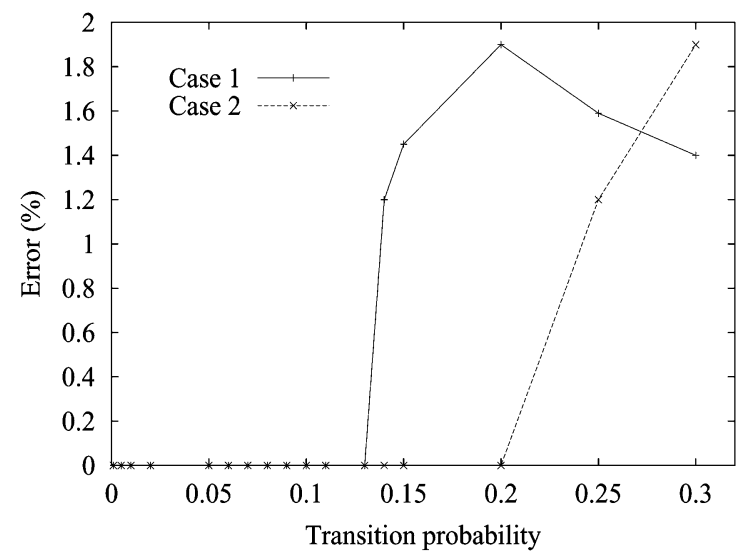

Fig. 9. The normalized approximation error $\left(\frac{D_{A}-D_{O}}{D_{O}} \times\right) 100 \%$ introduced due to the BD approximation in the computation of the minimum expected delay as a function of the transition probability $1-\delta$. In case $1, \gamma=\delta$. In case $2, \gamma=0.9$. Here, $G=Z=10, E V=100$ slots, and $K=5$.

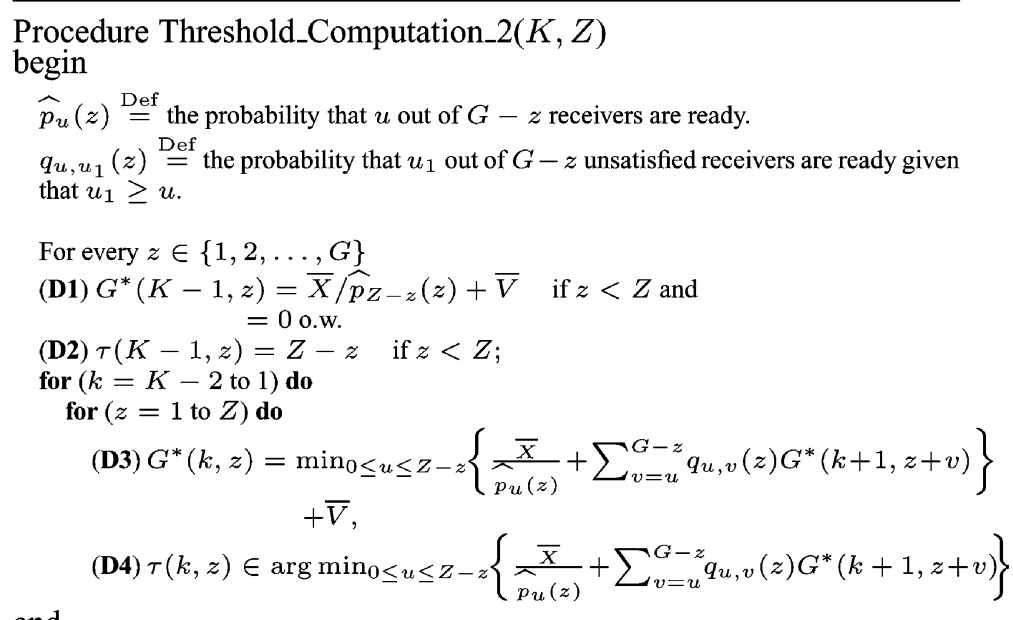

end

Procedure Transmission_Strategy_2 $(K, Z)$

begin

Initialize the system state $(k, z)=(0,0)$.

while $(z<Z)$ do

Transmit when the number of unsatisfied ready receivers (say $r$ ) is greater than or equal to $\tau(k, z)$.

Update the system state after transmission as follows: $k=k+1$ and $z=z+r$.

end

Fig. 10. Pseudocode for the optimal transmission policy, $\pi_{2}(K, Z)$, when the receiver readiness processes are i.i.d. Bernoulli. 


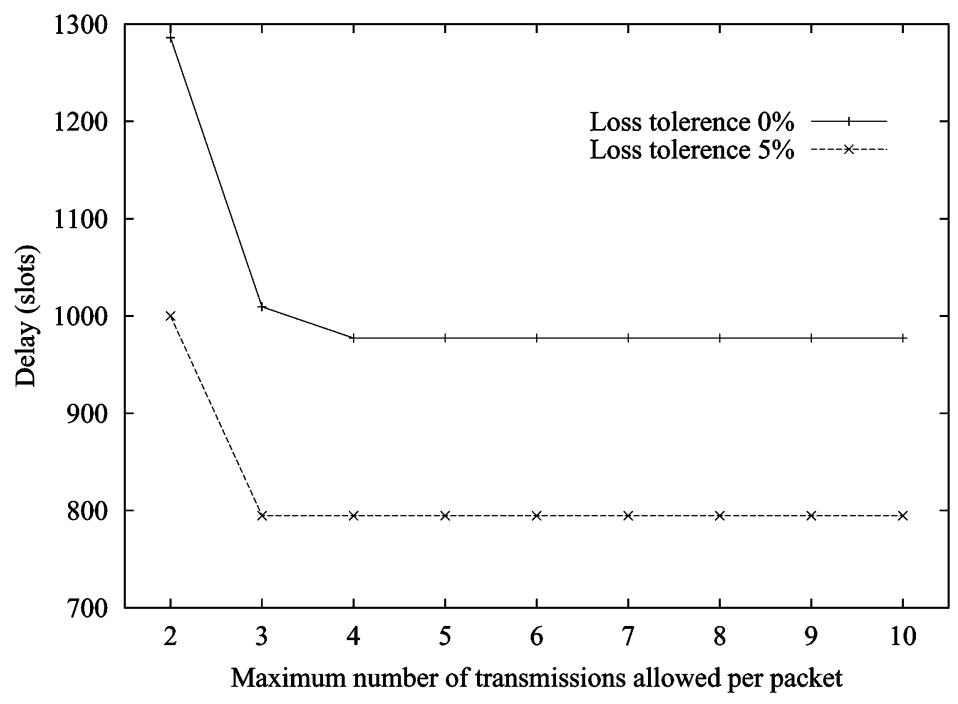

Fig. 11. The minimum expected delay when the readiness states are i.i.d. Markovian. Here, $E[V]=100, G=10$, and $\delta=\gamma=0.99$.

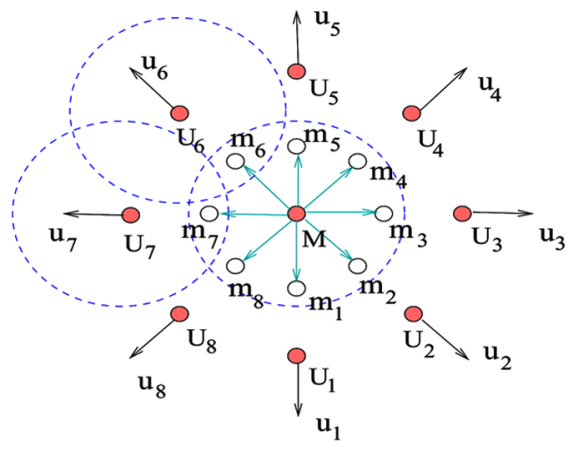

(a)

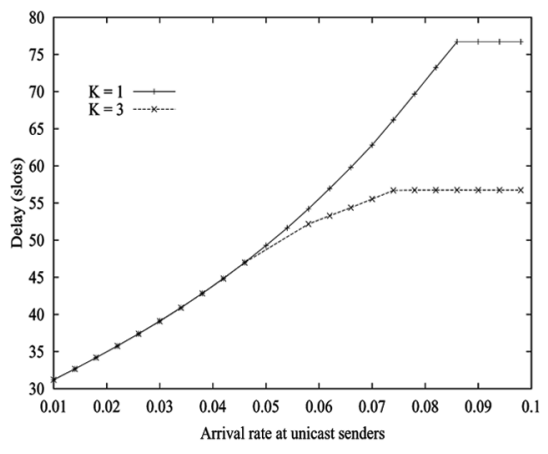

(b)

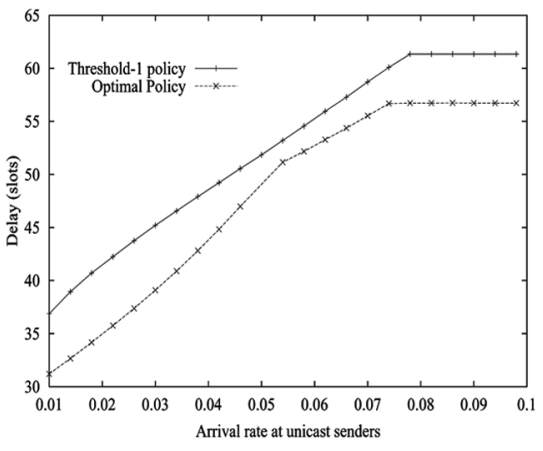

(c)

Fig. 12. (a) A topology we use to study the performance of the optimal policy using simulations. The topology has a multicast session with sender $M$ and eight receivers $m_{1}$ to $m_{8}$ and eight unicast sessions. Unicast session $i$ has sender $U_{i}$ and receiver $u_{i}, 1 \leq i \leq 8$. Each $U_{i}$ generates packets as per Poisson process with rate $\lambda_{U}$, and $M$ always has a packet and needs to deliver each packet to all the receivers. The mean packet sizes for $M$ and unicast senders is 20 and 1 slot, respectively. For every $i \in\{1, \ldots, 8\}, U_{i}$ is ready except when it is backing off or $m_{i}$ is receiving a packet. Also, $m_{i}$ is ready except when $U_{i}$ is transmitting a packet to $u_{i}$, and $M$ is not ready when it backs off, while $u_{i}$ is always ready. (b) The average delay of the multicast session for various values of $\lambda_{U}$. (c) The delays of the multicast session under optimal and threshold-1 policies.

retrieving a lost packet from another receiver is easier than retrieving a lost packet from the sender, e.g., when the distance between different receivers is significantly lower than that between the sender and any receiver. In such scenarios, the applications require upper bounds on the system loss. The policies presented in this correspondence guarantee that the system loss is upper-bounded by $G-Z$. When the receiver readiness process is i.i.d. Markovian, the policies also ensure that the loss at each receiver is upper-bounded by $(G-Z) / G$ with probability 1 . Thus, in these cases, $Z$ can, respectively, be determined from the system loss requirements and the loss tolerance at the individual receivers. When the receiver readiness processes are not i.i.d., loss guarantees can only be obtained for individual receivers by including explicit constraints related to such requirements in the Markov decision process (MDP) formulation. We expect that the complexity of solving such a constrained MDP will be exponential in $G$ and $K$ [1].

We now evaluate the performance of the proposed policies using numerical computations. Fig. 11 demonstrates that the expected delay significantly decreases as $K$ increases for small $K$, and saturates with further increase in $K$. Fig. 11 also shows that the minimum expected delay is significantly lower for $5 \%$ loss tolerance at each receiver (i.e., $100 *(G-Z) / G=5)$, than that for zero loss tolerance $(Z=G)$. Thus, a small number of transmissions and a small loss tolerance are usually sufficient to achieve the minimum expected delay.
We have so far assumed that the receiver readiness states are Markovian and are not affected by the transmission policies. Next, using simulations, we demonstrate that the resulting intuition and performance trends carry over to actual networks where these assumptions may not hold. We consider a simple symmetric topology shown in Fig. 12(a), where the readiness states are generated by packet transmissions. We model the readiness process for each receiver as a BD Markov process and estimate $\gamma$ and $\delta$ from the readiness states. We subsequently obtain the transmission policy using these estimates, $Z=G$, and the algorithm proposed in Fig. 7. In this simple example, the receiver readiness states generated by the above transmissions turn out to be ergodic. Thus, node $M$ can estimate the transition probabilities of the readiness states from observations, e.g., by updating the estimates of the transition rates every time it samples the readiness states of the receivers. Next, for moderate values of $\lambda_{U}$, like in Fig. 11, the proposed policy can significantly reduce the delay for multiple transmissions $(K=3)$ than when only one transmission $(K=1)$ is allowed (see Fig. 12(b)). Finally, we compare the performance of the proposed policy with a naive heuristic. In this heuristic, which we refer to as threshold-1 policy, $M$ transmits when a) at least one unsatisfied receiver is ready for the first $K-1$ transmissions and b) all the unsatisfied receivers are ready for the last transmission. The proposed policy achieves significantly smaller delay than the threshold-1 policy (see 
Fig. 12(c)). Proving that the receiver readiness states are ergodic and designing computationally tractable optimal policies in arbitrary networks constitute interesting problems for future research.

Finally, the computation time and storage requirements of the optimal policies $\pi(K, Z), \pi_{1}(K, Z), \pi_{2}(K, Z)$ are exponential in the input size in all cases, as the input size is $O(G)$ in the general case and $O(\log (G))$ when the receiver readiness states are i.i.d. Markovian. An interesting direction of future research is to determine whether the delay minimization problem is NP-hard.

\section{APPENDIX A \\ VALUE-ITERATION APPROACH}

We will prove the optimality results using the value-iteration approach that is used for solving the Bellman's equations [3]. We now describe the value iteration approach. Let $J_{l}(k, \vec{a}, \vec{j}), J_{l, T}(k, \vec{a}, \vec{j})$, and $J_{l, B}(k, \vec{a}, \vec{j})$ be defined iteratively as follows. For every $0 \leq k \leq$ $K-1$ and $\vec{a}, \vec{j} \in \mathcal{C}$ such that $\sum_{i=1}^{G} a_{i}<Z$

$$
\begin{aligned}
J_{l}(k, \vec{a}, \vec{j}) & =\min \left\{J_{l, T}(k, \vec{a}, \vec{j}), J_{l, B}(k, \vec{a}, \vec{j})\right\} \\
J_{l, T}(k, \vec{a}, \vec{j}) & =\bar{V}+\sum_{\vec{j}_{1} \in \mathcal{C}} B_{\vec{j}_{,}, \vec{j}_{1}} J_{l-1}\left(k+1, \vec{a} \circ \vec{j}, \vec{j}_{1}\right) \\
J_{l, B}(k, \vec{a}, \vec{j}) & =\bar{X}+\sum_{\vec{j}_{1} \in \mathcal{C}} B_{\vec{j}_{,} \vec{j}_{1}} J_{l-1}\left(k, \vec{a}, \vec{j}_{1}\right) .
\end{aligned}
$$

For termination states $(k, \vec{a}, \vec{j})\left(\sum_{i=1}^{G} a_{i} \geq Z\right)$

$$
J_{l}(k, \vec{a}, \vec{j})=J_{l, T}(k, \vec{a}, \vec{j})=J_{l, B}(k, \vec{a}, \vec{j})=0
$$

for every $l$. Moreover, $J_{l}(K, \vec{a}, \vec{j})=\infty$ if $\sum_{i=1}^{G} a_{i}<Z$ for every $l$.

For all $0 \leq k \leq K-1$,

$$
\begin{aligned}
& J_{0, T}(k, \vec{a}, \vec{j}), J_{0, B}(k, \vec{a}, \vec{j}), \quad \vec{a}, \vec{j} \in \mathcal{C} \\
& \lim _{l \rightarrow \infty} J_{l, T}(k, \vec{a}, \vec{j})=J_{T}^{*}(k, \vec{a}, \vec{j}) \\
& \lim _{l \rightarrow \infty} J_{l, B}(k, \vec{a}, \vec{j})=J_{B}^{*}(k, \vec{a}, \vec{j})
\end{aligned}
$$

and

$$
\lim _{l \rightarrow \infty} J_{l}(k, \vec{a}, \vec{j})=J^{*}(k, \vec{a}, \vec{j})
$$

(see [3, Proposition 2.1.2]).

\section{APPENDIX B}

\section{PROOF OF LEMMA 1}

Proof: Let states $(k, \vec{a}, \vec{j})$ and $\left(k, \vec{a}_{1}, \vec{j}_{1}\right)$ satisfy (5). If $z_{\vec{a}} \geq Z$, or if $k=K$ and $z_{\vec{a}}<Z$, then clearly $J^{*}(k, \vec{a}, \vec{j})=J^{*}\left(k, \vec{a}_{1}, \vec{j}_{1}\right)$. Thus, (6) follows. Now, let $k<K$ and $z_{\vec{a}}<Z$. Let $J_{0, T}(k, \vec{a}, \vec{j})=$ $J_{0, B}(k, \vec{a}, \vec{j})=0$ for all $0 \leq k \leq K-1$. We prove that

$$
J_{l}(k, \vec{a}, \vec{j})=J_{l}\left(k, \vec{a}_{1}, \vec{j}_{1}\right), \quad \forall l, k \in\{0, \ldots, K-1\}, z_{\vec{a}}<Z .
$$

Now, (6) follows after taking limits as $l$ goes to $\infty$ in (12).

To prove (12), it suffices to show the following for every $l$ :

$$
\begin{aligned}
& J_{l, B}(k, \vec{a}, \vec{j})=J_{l, B}\left(k, \vec{a}_{1}, \vec{j}_{1}\right) \\
& J_{l, T}(k, \vec{a}, \vec{j})=J_{l, T}\left(k, \vec{a}_{1}, \vec{j}_{1}\right) .
\end{aligned}
$$

We prove (13) and (14) using induction. Now, (13) and (14) clearly hold for $l=0$. We assume that (13) and (14) hold for every $l \leq L-1$, and prove them for $l=L$. Let $\mathcal{C}_{u}(\vec{a}, \vec{j})=\left\{\vec{j}^{\prime}: t_{\vec{a}, \vec{j}^{\prime}}=u\right\}$ for $u \in\left\{0, \ldots, G-z_{\vec{a}}\right\}$. Thus, from (11)

$$
\begin{aligned}
J_{L, B}(k, \vec{a}, \vec{j})= & \bar{X}+\sum_{u=0}^{G-z_{\vec{a}}} \sum_{\vec{j}^{\prime} \in \mathcal{C}_{u}(\vec{a}, \vec{j})} B_{\vec{j}, \vec{j}^{\prime}} J_{L-1}\left(k, \vec{a}, \vec{j}^{\prime}\right) . \\
J_{L, B}(k, \vec{a}, \vec{j})- & J_{L, B}\left(k, \vec{a}_{1}, \vec{j}_{1}\right) \\
= & \sum_{u=0}^{G-z_{\vec{a}}}\left[\sum_{\vec{j}^{\prime} \in \mathcal{C}_{u}(\vec{a}, \vec{j})} B_{\vec{j}^{\prime}, \vec{j}^{\prime}}-\sum_{\vec{j}^{\prime} \in \mathcal{C}_{u}\left(\vec{a}_{1}, \vec{j}_{1}\right)} B_{\vec{j}_{1}, \vec{j}^{\prime}}\right] \\
& \times J_{L-1}\left(k, \vec{a}, \vec{j}^{\prime}\right)(\text { from (5) and induction hypothesis). }
\end{aligned}
$$

Now

$$
\sum_{\vec{j}^{\prime} \in \mathcal{C}_{u}(\vec{a}, \vec{j})} B_{\vec{j}, \vec{j}^{\prime}}=\sum_{\vec{j}^{\prime} \in \mathcal{C}_{u}\left(\vec{a}_{1}, \vec{j}_{1}\right)} B_{\vec{j}_{1}, \vec{j}^{\prime}}
$$

for every $u \in\left\{0, \ldots, G-z_{\vec{a}}\right\}$ whenever the receiver readiness states are i.i.d. and (5) holds. Thus, $J_{L, B}(k, \vec{a}, \vec{j})=J_{L, B}\left(k, \vec{a}_{1}, \vec{j}_{1}\right)$. Hence, by induction, (13) follows. Equation (14) follows from similar arguments.

Henceforth, we denote the system state as $(k, z, t)$. Now, the Bellman's equations are as follows for $k \leq K-1$, $z<Z$, and $t \leq G-z$ :

$$
\begin{aligned}
& J^{*}(k, z, t)=\min \left\{J_{T}^{*}(k, z, t), J_{B}^{*}(k, z, t)\right\} \\
& J_{T}^{*}(k, z, t)=\bar{V}+\sum_{\widehat{t}=0}^{G-z-t} P_{0, \widehat{t}}(z+t) J^{*}(k+1, z+t, \widehat{t}) \\
& J_{B}^{*}(k, z, t)=\bar{X}+\sum_{\widehat{t}=0}^{G-z} P_{t, \widehat{t}}(z) J^{*}(k, z, \widehat{t}) .
\end{aligned}
$$

If $z \geq Z$, then as before $J^{*}(k, z, t)=0$ for every $k \leq K$. Also, $J^{*}(K, z, t)=\infty$ if $z<Z$. As described in the previous section, Bellman's equations can be solved using value iteration method. Let $J_{l}(k, z, t), J_{l, T}(k, z, t)$, and $J_{l, B}(k, z, t)$ be defined iteratively as follows for $0 \leq k \leq K-1,0 \leq z<Z$, and $0 \leq t \leq G-z$ :

$$
\begin{aligned}
J_{l}(k, z, t) & =\min \left\{J_{l, T}(k, z, t), J_{l, B}(k, z, t)\right\} \\
J_{l, T}(k, z, t) & =\bar{V}+\sum_{\widehat{t}=0}^{G-z-t} P_{0, \widehat{t}}(z+t) J_{l-1}(k+1, z+t, \widehat{t}) \\
J_{l, B}(k, z, t) & =\bar{X}+\sum_{\widehat{t}=0}^{G-z} P_{t, \widehat{t}}(z) J_{l-1}(k, z+t, \widehat{t}) .
\end{aligned}
$$

Also, $J_{l}(k, z, t)=0$ if $z \geq Z$ and $J_{l}(K, z, t)=\infty$ if $z<Z$ for every $l$.

\section{APPENDIX C}

PROOF OF THEOREM 1

We prove a supporting lemma (Lemma 2) which shows that $\mathcal{P}(K, G, Z, t)$ can be solved using a linear program LP2 which we describe next. Subsequently, we use Lemma 2 to prove Theorem 1.

LP2: Maximize $\sum_{k=0}^{K-1} \sum_{z=0}^{G} \sum_{t=0}^{G-z} \beta_{k, z, t} \widehat{J}(k, z, t)$

\section{Subject to:}

1) $\hat{J}(k, z, t)=0$ for every $z \geq Z, k \leq K-1$, and $t \leq G-z$,

2) $\widehat{J}(K-1, z, t)=\bar{V}+s_{t, Z-z}^{\prime}(z)$ for every $z<Z$,

3) $\widehat{J}(k, z, t) \leq \widehat{J}_{B}(k, z, t), k \leq K-1$, 
4) $\widehat{J}_{B}(k, z, t)=\bar{X}+\sum_{\widehat{t}=0}^{G-z} P_{t, \widehat{t}}(z) \widehat{J}(k, z, \widehat{t})$ for every $k \leq K-1$, $z \leq Z-1$, and $t \leq{ }^{t} \bar{G}-z$,

5) $\widehat{J}(k, z, t) \leq \widehat{J}_{T}(k, z, t), k \leq K-1$,

6) $\widehat{J}_{T}(k, z, t)=\bar{V}+\sum_{\widehat{t}=0}^{G-z-\bar{t}} P_{0, \widehat{t}}(z) \widehat{J}(k+1, z+t, \widehat{t})$ for every $k \leq K-1, z \leq Z-\overline{1}$, and $t \leq G-z$.

Let $\widehat{J}^{1}(k, z, t), \widehat{J}_{B}^{1}(k, z, t)$, and $\widehat{J}_{T}^{1}(k, z, t)$ denote the optimal solution of $\mathbf{L P 2}$ for every $k \leq K-1, z \in\{0, \ldots, G\}$, and $t \in$ $\{0, \ldots, G-z\}$.

Lemma 2: Let $\beta_{k, z, t} \geq 0$ for every $(k, z, t)$. Then, the following hold.

(A1) The linear program LP2 is always feasible.

(A2) If $\widehat{J}(k, z, t)$ is a feasible solution of LP2, then $J^{*}(k, z, t) \geq \widehat{J}(k, z, t)$ for every $(k, z, t)$.

(A3) If $\beta_{k, z, t}>0$, then $\widehat{J}^{1}(k, z, t)=J^{*}(k, z, t)$.

Proof: Note that the assignment $\widehat{J}(K-1, z, t)=\bar{V}+s_{t, Z-z}^{\prime}(z)$ for every $z<Z$, and $J(k, z, t)=0$ otherwise, is a feasible solution of LP2. Thus, (A1) follows. The proof for (A2) follows using arguments similar to those in [2, Ch. 7, pp. 376]. Next, $J^{*}(k, z, t)$ is a feasible solution of LP2. Thus, (A3) clearly follows from (A2).

Let $J^{\pi}(k, z, t)$ denote the expected termination time from state $(k, z, t)$ under policy $\pi$. Also, let $J_{T}^{\pi}(k, z, t)\left(J_{B}^{\pi}(k, z, t)\right.$, respectively) denote the expected termination time if the sender transmits (backs off, respectively) in $(k, z, t)$ and subsequent decisions are taken as per $\pi$.

\section{Proof of Theorem 1:}

Proof: If $z \geq Z$, then $J^{*}(k, z, t)$ is 0 for any $k, t$. Thus, (B1) in Fig. 5 obtains optimal termination times from every state $(k, z, t)$ such that $z \geq Z$. Thus, henceforth, we only consider $z<Z$.

We prove the following using induction on $k$.

(H1) For every $(k, z, t), \widehat{J}^{*}(k, z, t)=J^{*}(k, z, t)$.

For $k=K-1$, (H1) follows from (1). Now, we assume (H1) for $k>\widehat{k}$ and prove (H1) for $k=\widehat{k}$.

In LP2, we choose $\beta_{k, z, t}=1$ if $k=\widehat{k}$ and 0 otherwise. Thus,

$$
\widehat{J}^{1}(\widehat{k}, z, t)=J^{*}(\widehat{k}, z, t)(\text { from (A3) of Lemma } 2)
$$

Note that $\widehat{J}^{*}(\widehat{k}, z, t)$ is the optimal solution of LP1 in Fig. 5 for $k=\widehat{k}$. Now, LP1 in Fig. 5 for $k=\widehat{k}$ is similar to LP2 except that it has fewer constraints, and the right-hand sides of these constraints have $\widehat{J}^{*}(\widehat{k}+1, z, t)$ instead of $\widehat{J}(\widehat{k}+1, z, t)$. By induction hypothesis, $\widehat{J}^{*}(\widehat{k}+1, z, t)=J^{*}(\widehat{k}+1, z, t)$. Thus, from (A2) of Lemma 2 , the maximum value of the objective function of LP1 is greater than or equal to that of LP2. Thus,

$$
\sum_{z=0}^{G} \sum_{t=0}^{G-z} \widehat{J}^{*}(\widehat{k}, z, t) \geq \sum_{z=0}^{G} \sum_{t=0}^{G-z} \widehat{J}^{1}(\widehat{k}, z, t) .
$$

It can be easily seen that $J^{*}(k, z, t)$ for $k>\widehat{k}, \widehat{J}^{*}(\widehat{k}, z, t)$, and $\widehat{J}(k, z, t)=0$ for $k<\widehat{k}$ is a feasible solution for LP2. Thus,

$$
\widehat{J}^{*}(\widehat{k}, z, t) \leq J^{*}(\widehat{k}, z, t)(\text { from (A2) of Lemma } 2) .
$$

Thus, from (21)-(23), $\widehat{J}^{*}(\widehat{k}, z, t)=J^{1}(\widehat{k}, z, t)$. Now, from (21), (H1) holds for $k=\widehat{k}$.

The optimality of $\pi(K, Z)$ follows from (B3) and (H1).

\section{APPENDIX D \\ PROOFS OF THEOREMS 2 AND 3}

First, we derive some properties of the optimal solution (Lemmas 3 to 7), and using these we prove Theorems 2 and 3 . For any policy $\pi$

$$
J_{T}^{\pi}(k, z, u)=\bar{V}, \quad \text { if } u \geq Z-z .
$$

Let $\pi^{*}$ be the optimal policy.

Lemma 3: Let $z<Z, k<K$. Then, $\mathcal{T}_{k, z}$ is unique. If $u \geq Z-z$, $u \in \mathcal{T}_{k, z}$

Proof: Uniqueness of $\mathcal{T}_{k, z}$ follows since Bellman's equations (15)-(17) have unique solutions. Now, let $u \geq Z-z$. Since $z<$ $Z, k<K$, at least one more transmission is required to reach a termination state. Hence, $J^{*}(k, z, t) \geq \bar{V}$ for every $t$. Thus, from (17)

$$
\begin{aligned}
J_{B}^{*}(k, z, u) & \geq \bar{X}+\sum_{t=0}^{G-z} P_{u, t}(z) \bar{V} \\
& =\bar{X}+J_{T}^{*}(k, z, u)(\text { by }(24)) .
\end{aligned}
$$

Thus, $J_{B}^{*}(k, z, u)>J_{T}^{*}(k, z, u)$. Hence, $u \in \mathcal{T}_{k, z}$ by $(15)$

Thus, when $z<Z$ and $k<K, m_{k, z}$ is well defined.

Corollary 1: For $z<Z, \mathcal{T}_{K-1, z}=\{Z-z, \ldots, G-z\}$.

Proof: Let $u<Z-z$ and $z<Z$. Now, from (16) and since $J^{*}(K, z, t)=\infty$ for every $t, J_{T}^{*}(K-1, z, u)=\infty$. Thus, clearly, $J_{B}^{*}(K-1, z, u) \leq J_{T}^{*}(K-1, z, u)$. Thus, from (8), $u \notin \mathcal{T}_{K-1, z}$. The result follows from Lemma 3 .

Lemma 4: For every $k \leq K-1, z$, and $t \leq G-z, J^{*}(k, z, t) \leq$ $J^{*}(k+1, z, t)$.

Proof: If $z \geq Z$, then $J^{*}(k, z, t)=J^{*}(k+1, z, t)=0$. Thus, the lemma follows. Let $z<Z$ and $J_{0}(k, z, t)=0$ for every $k \leq$ $K-1, z<Z$, and $t \leq G-z$. We show that for every $l$

$$
J_{l}(k, z, t) \leq J_{l}(k+1, z, t), \quad \text { for all } k, z, t .
$$

Thus, the lemma follows after taking limits as $l$ goes to $\infty$ in (26).

Since $J_{l}(K, z, t)=\infty$ if $z<Z$ for every $l$, (26) holds for $l=0$. We assume that (26) holds for every $l<L$, and prove (26) for $l=L$. By induction hypothesis and (19)

$$
\begin{aligned}
J_{L, T}(k+1, z, t) \geq & \bar{V}+\sum_{t_{1}=0}^{G-z-t} P_{t, t_{1}}(z+t) J_{L-1}\left(k+1, z+t, t_{1}\right) \\
= & J_{L, T}(k, z, t) \quad(\text { from }(19)) . \\
J_{L, B}(k+1, z, t) \geq & \bar{X}+\sum_{t_{1}=0}^{G-z} P_{t, t_{1}}(z) J_{L-1}\left(k, z, t_{1}\right) \\
& (\text { by induction hypothesis and (20)) } \\
= & J_{L, B}(k, z, t)(\text { from }(20)) . \\
J_{L}(k+1, z, t) \geq & \min \left\{J_{L, T}(k, z, t), J_{L, B}(k, z, t)\right\} \\
& (\text { from }(18),(27) \text { and }(28)) . \\
= & J_{L}(k, z, t)(\text { from }(18)) .
\end{aligned}
$$

Lemma 5: Let $k<K$ and $z<Z$. Then, $m_{k, z}>0$. 
Proof: From (16) and since $\bar{V} \geq \bar{X}$

$$
\begin{aligned}
J_{T}^{*}(k, z, 0) & \geq \bar{X}+\sum_{t=0}^{G-z} P_{0, t}(z) J^{*}(k+1, z, t) \\
& \geq \bar{X}+\sum_{t=0}^{G-z} P_{0, t}(z) J^{*}(k, z, t)(\text { by Lemma } 4) \\
& =J_{B}^{*}(k, z, 0)(\text { from }(17)) .
\end{aligned}
$$

Thus, from (8), $0 \notin \mathcal{T}_{k, z}$, for all $k, z$. The result follows.

$$
\text { Let } \mathcal{S}_{k, z}=\left\{u: P_{0, u}(z)>0\right\} \text { if } k>0 \text { and } \mathcal{S}_{0, z}=\{0, \ldots, G\} \text {. }
$$

Lemma 6: For any policy $\pi$, if $J^{\pi}(k+1, \widehat{z}, u)=J^{*}(k+1, \widehat{z}, u)$ for every $\widehat{z}$ and $u \in \mathcal{S}_{k+1, \widehat{z}}$, then for every $z<Z$ and $t \leq G-z$

$$
J_{T}^{\pi}(k, z, t)=J_{T}^{*}(k, z, t) .
$$

Proof: Note that

$$
J_{T}^{\pi}(k, z, t)=\bar{V}+\sum_{u=0}^{G-z-t} P_{0, u}(z+t) J^{\pi}(k+1, z+t, u) .
$$

Thus, the result follows from the condition given in the lemma and (16).

Now we define some additional notations. Let $\mathcal{A}(z)=\{0, \ldots, G-$ $z\}$. Note that $\mathcal{A}(z)$ is the state space of aggregate readiness process of $G-z$ receivers. Consider a set $A \subseteq \mathcal{A}(z)$. Let $r_{v, u}(A)$ denote the probability that the first state visited in $A$ is $u$ starting from state $v$ in the aggregate readiness process of $G-z$ receivers. Also, let $x_{v}(A)$ denote the product of $\bar{X}$ and the expected number of sample points required to reach any of the states $u \in A$ for the first time starting from state $v$ in the aggregate readiness process of $G-z$ receivers.

Any policy needs to transmit at least once more from a state $(k, z, t)$ for $k<K, z<Z$. Hence, if $k<K, z<Z$

$$
\begin{aligned}
J^{*}(k, z, t) & =\min _{A \subseteq \mathcal{A}(z)}\left\{x_{t}(A)+\sum_{u \in A} r_{t, u}(A) J_{T}^{*}(k, z, u)\right\} \\
& =x_{t}\left(\mathcal{T}_{k, z}\right)+\sum_{u \in \mathcal{T}_{k, z}} r_{t, u}\left(\mathcal{T}_{k, z}\right) J_{T}^{*}(k, z, u) .
\end{aligned}
$$

Lemma 7: Let the aggregate readiness process of the unsatisfied receivers be a $\mathrm{BD}$ process and $z<Z, k<K, t \leq m_{k, z}$

$$
\begin{aligned}
J^{\pi^{*}}(k, z, t) & =s_{t, m_{k}, z}(z)+J_{T}^{\pi^{*}}\left(k, z, m_{k, z}\right) \\
& =\min _{u \geq t}\left\{s_{t, u}(z)+J_{T}^{\pi^{*}}(k, z, u)\right\} .
\end{aligned}
$$

Proof: Since the aggregate readiness process of unsatisfied receivers is $\mathrm{BD}$ and $t \leq m_{k, z}$

$$
\begin{aligned}
x_{t}\left(\mathcal{T}_{k, z}\right) & =s_{t, m_{k, z}}(z) \quad \text { and } \\
r_{t, u}\left(\mathcal{T}_{k, z}\right) & =1, \quad \text { for } u=m_{k, z} \\
& =0, \quad \text { o.w. }
\end{aligned}
$$

Now, (31) follows from (30).

From (31), since $m_{k, z} \geq t$

$$
J^{\pi^{*}}(k, z, t) \geq \min _{u \geq t}\left\{s_{t, u}(z)+J_{T}^{\pi^{*}}(k, z, u)\right\} .
$$

From (29)

$$
J^{\pi^{*}}(k, z, t) \leq \min _{u \geq t}\left\{s_{t, u}(z)+J_{T}^{\pi^{*}}(k, z, u)\right\}
$$

Thus, (32) follows.

Corollary 2: Let the aggregate readiness process of the unsatisfied receivers be a BD process. Then, if $0<k<K, z<Z$, and $t \in \mathcal{S}_{k, z}$

$$
\begin{aligned}
J^{\pi^{*}}(k, z, t) & =s_{t, m_{k, z}}(z)+J_{T}^{\pi^{*}}\left(k, z, m_{k, z}\right) \\
& =\min _{u \geq 1}\left\{s_{t, u}(z)+J_{T}^{\pi^{*}}(k, z, u)\right\} .
\end{aligned}
$$

Proof: Since the aggregate readiness process of unsatisfied receivers is a BD process, $\mathcal{S}_{k, z}=\{0,1\}$ for every $k>0$ and $z<Z$. Thus, by Lemma $5, t \leq m_{k, z}$. Thus, (33) follows from Lemma 7 . Using similar arguments as in the proof of (32) from (31), it can be shown that (34) follows from (33).

\section{Proof of Theorem 2:}

Proof: Let $\widehat{J}^{*}(k, z, t)$ be as defined in Fig. 7. We show that for every $k \leq K-1, z$, and $t \in \mathcal{S}_{k, z}$

$$
\widehat{J}^{*}(k, z, t)=J^{\pi_{1}(K, Z)}(k, z, t)=J^{\pi^{*}}(k, z, t) .
$$

Let $z \geq Z$. Clearly, $J^{\pi}(k, z, t)=J^{\pi^{*}}(k, z, t)=0$, for every $\pi$. Thus, (35) follows from (C1) in Fig. 7. Thus, henceforth, we consider the case $z<Z$. Also, when $K=1$, clearly $\pi(K, Z)$ is optimal. Thus, henceforth, we consider the case $K>1$.

By (C1) and (C2) in Fig. 7, $\widehat{J}^{*}(K-1, z, t)=J^{\pi_{1}(K, Z)}(K-1, z, t)$. Now, from Corollary $1, m_{K-1, z}=Z-z$. Moreover, for $k>0$, $\mathcal{S}_{k, z}=\{0,1\}$ since the aggregate readiness process is a BD process. Hence, for any $t \in \mathcal{S}_{K-1, z}, t \leq Z-z$. Thus, $\pi^{*}$ will transmit when $Z-z$ unsatisfied receivers are ready. From $(\mathbf{C 2})$ in Fig. $7, \pi_{1}(K, Z)$ also transmits only when $Z-z$ unsatisfied receivers are ready. Thus, (35) holds.

We assume that (35) holds for all $\widehat{k} \in\{K-1, \ldots, k+1\}$, and prove (35) for $k$. First, for any policy $\pi$ and $z<Z$

$$
\begin{aligned}
J_{T}^{\pi}(k, z, t)=\bar{V}+\left(1-\alpha_{0}(z)\right) J^{\pi}(k+1, z+t, 1) \\
\\
+\alpha_{0}(z) J^{\pi}(k+1, z+t, 0) .
\end{aligned}
$$

We first consider $k \geq 1$. From (C0) in Fig. 7, (36), Lemma 6, and the induction hypothesis, for every $u$

$$
\begin{aligned}
\widehat{J}_{T}^{*}(k, z, u) & =J_{T}^{\pi_{1}(K, Z)}(k, z, u)=J_{T}^{\pi^{*}}(k, z, u) . \\
\text { Now, } J^{\pi_{1}(K, Z)}(k, z, t) & =s_{t, \tau(k, z)}(z)+J_{T}^{\pi_{1}(K, Z)}(k, z, \tau(k, z)) .
\end{aligned}
$$

Thus, from (C4), (C5), (37), and (38), for every $t \in \mathcal{S}_{k, z}$

$$
\widehat{J}^{*}(k, z, t)=J^{\pi_{1}(K, Z)}(k, z, t) .
$$


Now, for every $t \in \mathcal{S}_{k, z}$

$$
\begin{aligned}
& J^{\pi_{1}(K, Z)}(k, z, t) \\
& =s_{t, \tau(k, z)}(z)+\widehat{J}_{T}^{*}(k, z, \tau(k, z)) \quad(\text { from }(37) \text { and (38)) } \\
& =s_{t, 1}(z)+s_{1, \tau(k, z)}(z)+\widehat{J}_{T}^{*}(k, z, \tau(k, z)) \\
& \quad(\text { since } \tau(k, z) \geq 1) \\
& =s_{t, 1}(z)+\min _{1 \leq u \leq Z-z}\left\{\begin{array}{c}
\left.s_{1, u}(z)+\widehat{J}_{T}^{*}(k, z, u)\right\} \\
\quad(\text { by }(\mathbf{C 3}) \text { in Fig. 7) }
\end{array}\right. \\
& =\min _{1 \leq u \leq Z-z}\left\{s_{t, u}(z)+J_{T}^{\pi^{*}}(k, z, u)\right\}(\text { by (37)). }
\end{aligned}
$$

Now, we show that for every $u>Z-z$ and $t \in \mathcal{S}_{k, z}$

$$
s_{t, Z-z}(z)+J_{T}^{\pi^{*}}(k, z, Z-z) \leq s_{t, u}(z)+J_{T}^{\pi^{*}}(k, z, u) .
$$

Since $t \in \mathcal{S}_{k, z}, t \leq Z-z$. Since aggregate readiness process of the unsatisfied receivers is a BD process, for every $u>Z-z$

$$
\begin{aligned}
s_{t, u}(z) & =s_{t, Z-z}(z)+s_{Z-z, u}(z), \\
J_{T}^{\pi^{*}}(k, z, u) & =J_{T}^{\pi^{*}}(k, z, Z-z)=\bar{V} \quad(\text { from (24)). }
\end{aligned}
$$

Now, (41) follows from (42) and (43). Thus, from (40) and (41)

$$
\begin{aligned}
J^{\pi_{1}(K, Z)}(k, z, t) & =\min _{u \geq 1}\left\{s_{t, u}(z)+J_{T}^{\pi^{*}}(k, z, u)\right\} \\
& \left.=J^{\pi^{*}}(k, z, t) \text { (by Corollary } 2\right) .
\end{aligned}
$$

Thus, from (39) and (44), (35) hold for $k \geq 1$.

Now, we prove (35) for $k=0$. Thus, from (C0) in Fig. 7, (36), Lemma 6, and the induction hypothesis, for every $u$

$$
\widehat{J}_{T}^{*}(0,0, u)=J_{T}^{\pi_{1}}(0,0, u)=J_{T}^{\pi^{*}}(0,0, u) .
$$

We consider two cases. First, $m_{0,0} \geq t$ or $t \in \mathcal{T}_{0,0}$. Second, $m_{0,0}<$ $t$ and $t \notin \mathcal{T}_{0,0}$. In the first case, similar to the proof in Lemma 7, it can be shown that

$$
\begin{aligned}
J^{\pi^{*}}(0,0, t) & =\min _{u \geq t}\left\{s_{t, u}(z)+J_{T}^{\pi^{*}}(0,0, u)\right\} \\
& =J_{1}(0,0, t) \text { (Fig. 7) (by (45)). }
\end{aligned}
$$

Now, in the second case, let $m_{0,0}^{1}=\max _{u<t}\left\{u: u \in \mathcal{T}_{0,0}\right\}$, and $m_{0,0}^{2}=\min _{u>t}\left\{u: u \in \mathcal{T}_{0,0}\right\}$. Since the aggregate readiness process is a BD process, and $\pi^{*}$ transmits when the system reaches a state in $\mathcal{T}_{0,0}, \pi^{*}$ will transmit in $m_{0,0}^{1}$ or $m_{0,0}^{2}$. Thus, by (8)

$$
\begin{aligned}
J^{\pi^{*}}(0,0, t) & =s_{t, m_{0,0}^{1}} \| m_{0,0}^{2}(0)+\sum_{u=1}^{2} r_{t, m_{0,0}^{u}} J_{T}^{\pi^{*}}\left(0,0, m_{0,0}^{u}\right) \\
& \geq \min _{\substack{v_{1}<t \\
v_{2}>t}}\left\{s_{t, v_{1} \| v_{2}}(0)+\sum_{u=1}^{2} r_{t, v_{u}} J_{T}^{\pi^{*}}\left(0,0, v_{u}\right)\right\} \\
& =J_{2}(0,0, t) \text { as defined in Fig. } 7 \text { (by (45)). }
\end{aligned}
$$

From (29), $J^{\pi^{*}}(0,0, t) \leq J_{2}(0,0, t)$. Thus,

$$
J^{\pi^{*}}(0,0, t)=J_{2}(0,0, t) .
$$

If case 1 holds, then by (46) and (29), $J_{1}(0,0, t) \leq J_{2}(0,0, t)$. If case 2 holds, from (47) and (29), $J_{1}(0,0, t) \geq J_{2}(0,0, t)$. Thus, $J^{\pi^{*}}(0,0, t)=\min \left\{J_{1}(0,0, t), J_{2}(0,0, t)\right\}$ for every $t$. Thus, (35) follows from (45), (C6) and (C7) in Fig. 7. The result follows.

\section{Proof for Theorem 3:}

Proof: We first show that the optimal policy is threshold type, i.e., if $t \in \mathcal{T}_{k, z}$, then $u \in \mathcal{T}_{k, z}$ for every $u \geq t$. Let $J_{0, T}(k, z, t)=$ $J_{0, B}(k, z, t)=0$ if $k<K$. We prove that for each iteration $l$, for every $k \leq K-1, z<Z$. and $t \leq G-z$

(H1) if $J_{l, T}(k, z, t)<J_{l, B}(k, z, t)$, then $J_{l, T}(k, z, t+1)<$ $J_{l, B}(k, z, t+1)$

(H2) $\quad J_{l}(k, z+1, t) \leq J_{l}(k, z, t)$, and

(H3) $J_{l}(k, z+1, t-1) \leq J_{l}(k, z, t)$.

Clearly, (H1), (H2), and (H3) hold for $l=0$. We assume that (H1), (H2), and (H3) hold till the $l$ th iteration, and prove these in the $l+1$ th iteration.

Let $p_{i}(z)$ be the probability that $i$ out of $G-z$ unsatisfied receivers are ready. Then

$$
\begin{aligned}
J_{l+1, B}(k, z, t) & =\bar{X}+\sum_{i=0}^{G-z} p_{i}(z) J_{l}(k, z, i)(\text { by }(20)) \\
& =J_{l+1, B}(k, z, t+1) .
\end{aligned}
$$

From (19)

$$
\begin{aligned}
& J_{l+1, T}(k, z, t+1) \\
& \quad=\bar{V}+\sum_{i=0}^{G-z-t-1} p_{i}(z+t+1) J_{l}(k+1, z+t+1, i) .
\end{aligned}
$$

Now

$$
\begin{aligned}
J_{l+1, T}(k, z, t)= & \bar{V}+\sum_{i=0}^{G-z-t} p_{i}(z+t) J_{l}(k+1, z+t, i) \\
= & \bar{V}+\sum_{i=0}^{G-z-t-1} p_{i}(z+t+1) p J_{l} \\
& \times(k+1, z+t, i+1) \\
& +\sum_{i=0}^{G-z-t-1} p_{i}(z+t+1)(1-p) J_{l} \\
& \times(k+1, z+t, i)
\end{aligned}
$$

(since the receiver readiness states are i.i.d.).

By induction hypotheses (H2) and (H3) and since $0 \leq p \leq 1$

$$
\begin{aligned}
& J_{l}(k+1, z+t+1, i) \\
& \quad \leq p J_{l}(k+1, z+t, i+1)+(1-p) J_{l}(k+1, z+t, i) .
\end{aligned}
$$

From (49), (51), and (52)

$$
J_{l+1, T}(k, z, t) \geq J_{l+1, T}(k, z, t+1) \text {. }
$$

Similarly, it can be shown that

$$
J_{l+1, B}(k, z+1, t) \leq J_{l+1, B}(k, z, t) .
$$

Proof of statement (H1): Let $J_{l+1, T}(k, z, t)<J_{l+1, B}(k, z, t)$. Then, from (53)

$$
\begin{aligned}
J_{l+1, T}(k, z, t+1) & <J_{l+1, B}(k, z, t) \\
& =J_{l+1, B}(k, z, t+1)(\text { by }(48)) .
\end{aligned}
$$

Thus, (H1) holds for iteration $l+1$. 
Proof of statement (H2): From (50)

$$
J_{l+1, T}(k, z+1, t)=J_{l+1, T}(k, z, t+1) .
$$

Thus, from (53), $J_{l+1, T}(k, z+1, t) \leq J_{l+1, T}(k, z, t)$. Then, (H2) follows from (18) and (54).

Proof of statement (H3): From (48) and (54)

$$
J_{l+1, B}(k, z+1, t-1) \leq J_{l+1, B}(k, z, t) .
$$

From (55)

$$
J_{l+1, T}(k, z+1, t-1) \leq J_{l+1, T}(k, z, t) .
$$

Then, (H3) follows from (18).

Thus, (H1), (H2), and (H3) hold for all $l$.

After taking limits as $l$ goes to $\infty$ in (H1), it follows that the optimal policy is threshold type.

Now, we show that the algorithm in Fig. 10 obtains a threshold that minimizes the expected termination time for every $k \leq K$ and $z<Z$. Let $G^{\pi}(k, z)$ denote the expected time to terminate under a policy $\pi$ after the $k$ th transmission and the subsequent backoff, if $z$ receivers are satisfied after $k$ transmissions.

We show that for every $k \leq K-1$ and $z$

$$
G^{*}(k, z)=G^{\pi^{*}}(k, z) .
$$

Since $G^{*}(k, z)=G^{\pi_{2}(K, Z)}(k, z),(56)$ proves the optimality of $\pi_{2}(K, Z)$.

Note that if $z \geq Z$, then $G^{*}(k, z)=G^{\pi^{*}}(k, z)=0$ for every $k$. Thus, (56) follows. Henceforth, we consider $z<Z$.

Let $k=K-1$. Clearly, $\pi^{*}$ transmits when at least $Z-z$ unsatisfied receivers are ready. Thus, $G^{\pi^{*}}(K-1, z)=\frac{\bar{X}}{\widehat{p}_{Z-z}}+\bar{V}$ where $\left\{\widehat{p}_{u}\right\}$ are as defined in Fig. 10. Thus, (56) follows.

Now, we assume (56) for every $\widehat{k}>k$ and show (56) for $k$. Clearly

$G^{\pi^{*}}(k, z)=\frac{\bar{X}}{\widehat{p}_{m_{k, z}}}+\bar{V}+\sum_{v=m_{k, z}}^{G-z-v} q_{m_{k, z}, v}(z+v) G^{\pi^{*}}(k+1, z+v)$

where $\left\{q_{u, v}(z)\right\}$ are as defined in Fig. 10. Now, from Lemmas 3 and 5, $0<m_{k, z} \leq Z-z$. Thus, from (57)

$$
\begin{aligned}
& G^{\pi^{*}}(k, z) \\
& \geq \min _{1 \leq u \leq Z-z}\left\{\frac{\bar{X}}{\widehat{p}_{u}}+\bar{V}+\sum_{v=u}^{G-z-v} q_{u, v}(z+v) G^{\pi^{*}}(k+1, z+v)\right\} \\
& =\min _{1 \leq u \leq Z-z}\left\{\frac{\bar{X}}{\widehat{p}_{u}}+\bar{V}+\sum_{v=u}^{G-z-v} q_{u, v}(z+v) G^{*}(k+1, z+v)\right\}
\end{aligned}
$$

(from induction hypothesis).

Clearly

$$
G^{\pi^{*}}(k, z) \leq G^{\pi_{2}(K, Z)}(k, z)=G^{*}(k, z) .
$$

Thus, $G^{\pi^{*}}(k, z)=G^{*}(k, z)$. The result follows.

\section{REFERENCES}

[1] E. Altman, Constrained Markov Decision Processes. London, U.K.: Chapman \& Hall/CRC, 1998, vol. 1.

[2] D. P. Bertsekas, Dynamic Programming and Optimal Control, 2nd ed. Belmont, MA: Athena Scientific, 2000, vol. 1.

[3] — Dynamic Programming and Optimal Control. Belmont, MA: Athena Scientific, 2001, vol. 2.
[4] J. Byers, M. Luby, M. Mitzenmacher, and A. Rege, "A digital fountain approach to reliable distribution of bulk data," in Proc. ACM SIGCOMM, Vancouver, BC, Canada, Sep. 1998, pp. 56-67.

[5] M. Gerla, C.-C. Chiang, and L. Zhang, "Tree multicast strategies in mobile, multihop wireless networks," ACM/Baltzer J. Mobile Networks and Applications (MONET), vol. 4, no. 3, pp. 193-207, 1999.

[6] N. Karmarkar, "A new polynomial-time algorithm for linear programming," Combinatorica, vol. 4, no. 4, pp. 373-395, 1984.

[7] J. Kurose and K. Ross, Computer Networking: A Top-Down Approach Featuring the Internet, 2 ed. Reading, MA: Addison-Welsley/Longman, 2003.

[8] E. Pagani and G. Rossi, "Reliable broadcast in mobile multihop packet networks," in Proc. Int. Conf. Mobile Computing and Networking (MOBICOM), Budapest, Hungary, Sep. 1997.

[9] S. Shakkottai and R. Srikant, "Scheduling real time traffic with deadlines over a wireless channel," ACM/Baltzer Wireless Netw. J., vol. 8, no. 1 , pp. 13-26, 2002

[10] K. Tang and M. Gerla, "Random access mac for efficient broadcast support in ad hoc networks," in Proc. IEEE Wireless Communications and Networking Conf. (WCNC), Chicago, IL, Sep. 2000, pp. 454-459.

[11] _ _ "Mac reliable broadcast in ad hoc networks," in Proc. Military Communications Conf. (MILCOM), Washington, DC, Oct. 2001, pp. $1008-1013$

[12] J. E. Wieselthier, G. D. Nguyen, and A. Ephremides, "On the construction of energy-efficient broadcast and multicast trees in wireless networks," in Proc. IEEE INFOCOM, Conf. Computer Communications, Tel-Aviv, Israel, Mar. 2000, pp. 585-594.

\section{Unitary Space-Time Constellation Analysis: An Upper Bound for the Diversity}

Guangyue Han and Joachim Rosenthal, Senior Member, IEEE

Abstract-The diversity product and the diversity sum are two very important parameters for a good-performing unitary space-time constellation. A basic question is what the maximal diversity product (or sum) is. In this correspondence, we are going to derive general upper bounds on the diversity sum and the diversity product for unitary constellations of any dimension $n$ and any size $m$ using packing techniques on the compact Lie group $U(n)$.

Index Terms-Diversity product, diversity sum, multiple antennas, space-time coding, space-time constellations.

\section{INTRODUCTION}

Let $A$ be a matrix with complex entries and $A^{*}$ denote the conjugate transpose of $A$. Let $\|\cdot\|$ denote the Frobenius norm of a matrix, i.e.,

$$
\|A\|=\sqrt{\operatorname{tr}\left(A A^{*}\right)} .
$$

A square matrix $A$ is called unitary if $A^{*} A=A A^{*}=I$, where $I$ denotes the identity matrix. We denote by $U(n)$ the set of all $n \times n$

Manuscript received January 5, 2004. This work was supported in part by the National Science Foundation under Grants DMS-00-72383 and CCR-02-05310. The work of G. Han was also supported by a fellowship from the Center of Applied Mathematics at the University of Notre Dame. The work of J. Rosenthal was also supported by the Swiss National Science Foundation under Grant 107887. This research was initiated while the authors were visiting the Institute Mittag-Leffler, Stockholm, Sweden, in May 2003. The hospitality and the financial support of the Institute Mittag-Leffler are gratefully acknowledged.

G. Han is with the Department of Mathematics, The University of British Columbia, Vancouver, BC V6T 1Z2, Canada (e-mail: ghan@math.ubc.ca).

J. Rosenthal is with the Department of Mathematics, University of Zürich, CH-8057 Zürich, Switzerland (e-mail: rosen@math.unizh.ch).

Communicated by B. Hassibi, Associate Editor for Communications.

Digital Object Identifier 10.1109/TIT.2006.881754 